\title{
Mixed Dynamics in a Parabolic Standard Map
}

\author{
L. M. Lerman* \\ Faculty of Mathematics \& Mechanics \\ Lobachevsky State University of Nizhny Novgorod, \\ Nizhny Novgorod, 603950 Russia \\ lermanl@mm.unn.ru
}

\author{
J. D. Meiss ${ }^{\dagger}$ \\ Department of Applied Mathematics \\ University of Colorado \\ Boulder, CO 80309-0526 \\ James.Meiss@colorado.edu
}

August 27, 2015

\begin{abstract}
We use numerical and analytical tools to provide arguments in favor of the existence of a family of smooth, symplectic diffeomorphisms of the two-dimensional torus that have both a positive measure set with positive Lyapunov exponent and a positive measure set with zero Lyapunov exponent. The family we study is the unfolding of an almost-hyperbolic diffeomorphism on the boundary of the set of Anosov diffeomorphisms, proposed by Lewowicz.
\end{abstract}

\section{Introduction}

Due to an extremely complicated intermixture of regular and chaotic orbits, the problem of the orbit structure of a generic, smooth symplectic map remains mainly open, even for the two-dimensional case. When the map is sufficiently smooth, its phase space typically exhibits both regular dynamics due to invariant KAM curves (for instance, in the neighborhood of elliptic periodic orbits) and seas of chaotic orbits (which numerical investigations indicate can be densely covered by a single orbit). Moreover, such structures are observed - again in numerical simulations - to occur at all scales. All this is well known and shown in many papers, for a review see, e.g., [Mei92]. It is generally agreed that no tools currently exist that allow one to rigorously elucidate the main points of this observed picture [Sin95]. Of course, selected parts of this landscape can be explained; for example, KAM theory provides a proof of the existence of invariant curves near generic elliptic periodic points, and it is known that a generic homoclinic bifurcation gives rise to a transitive set of full Hausdorff dimension [Gor12]. However even for this case, there is essentially no rigorous measure-theoretic

${ }^{*}$ LML was supported in part by RFBR (grant 14-01-00344a), the Russian Ministry of Science and Education (project 1.1410.2014/K, target part) and the Russian Science Foundation (project 14-41-00044). Useful conversations with V. Grines are gratefully acknowledged. Both authors acknowledge a support from CRDF (grant FSAX-14-60273$0)$.

${ }^{\dagger}$ JDM was supported in part by NSF grant DMS-1211350. The suggestion of Robert Easton for the construction in App. C is gratefully acknowledged.

(C) 2015. This manuscript version is made available under the Elsevier user license http://www.elsevier.com/open-access/userlicense/1.0/ 
characterization of the orbit behavior in the so-called chaotic zones - as depicted in Arnold's famous sketch [Arn63, Ber78].

There has been much study of the destruction of invariant curves, and the resulting transition from regular (quasiperiodic) to irregular (chaotic) behavior, in parameterized families of areapreserving maps. Since a smooth invariant curve is not isolated, its destruction is caused by a loss of smoothness and, at least for twist maps, to the formation of a new, quasiperiodic invariant Cantor set: an Aubry-Mather set [ALD83, Mat82]. In many families, one observes the ultimate destruction of all the invariant circles (of a given homotopy class), and this leads to the study of the "last" invariant curve, and the development of Greene's residue criterion and renormalization theory [Mac93].

At the opposite extreme, the ergodicity and hyperbolicity properties of Anosov diffeomorphisms are well-understood [Fra70]. This extreme of uniform hyperbolicity can be thought of as a complementary limit to integrability: the study of perturbations from "anti-integrability" was initiated in [AA90]. Aubry's results are based on the consideration of infinitely-degenerate diffeomorphisms and provide proofs of the existence of horseshoes; however, they do not lead to proofs of a positive measure of chaotic orbits.

There have been attempts to understand the dynamics of symplectic diffeomorphisms on the torus beyond the boundary of the Anosov maps [Prz82]. Przytycki proved the existence of a curve of diffeomorphisms that cross the Anosov boundary such that, outside the boundary, there is a domain on the torus bounded by a heteroclinic cycle formed by merged separatrices of two saddles that contains a generic elliptic fixed point. The remaining set of positive measure has a nonhyperbolic structure and positive Lyapunov exponent. The drawback of this example is in its infinite codimension in the space of smooth symplectic diffeomorphisms with $C^{5}$-topology: the merging of separatrices of saddles is a codimension-infinity phenomenon. Przytycki's family unfolds a smooth, almost-hyperbolic symplectic diffeomorphism of the torus proposed earlier by Lewowicz [Lew80]. This diffeomorphism is a K-system that has positive Lyapunov exponent [CE01].

This same trick (with the same drawbacks) was used later in [Liv04] to construct symplectic diffeomorphisms arbitrarily close to Lewowicz's almost-hyperbolic map. Smooth symplectic, transitive diffeomorphisms that are K-systems on closed two-dimensional manifolds other than tori were constructed in [Kat79] (see also, [GK82]). Again it is not clear how these results can be used to understand the orbit structure for a generic diffeomorphism.

Following [Ler10], we study the map $f: \mathbb{T}^{2} \rightarrow \mathbb{T}^{2}$, where $\mathbb{T}=\mathbb{R} / \mathbb{Z}$, defined through

$$
f(x, y)=(x+y+g(x), y+g(x)) \bmod 1 .
$$

We will use the fundamental domain $\left[-\frac{1}{2}, \frac{1}{2}\right)^{2}$ for the torus, so e.g., $x \bmod 1=x-\left\lfloor x+\frac{1}{2}\right\rfloor$. If the "force" $g$ were a degree-zero circle map, then (1) would be a generalized Chirikov standard map [Mei92]. Instead, we assume that $g$ is a degree-one, circle map:

$$
g(x+1)=g(x)+1 .
$$

When $g$ is a monotone increasing diffeomorphism, (1) is Anosov: every orbit is uniformly hyperbolic and $f$ is topologically conjugate to Arnold's cat map $a: \mathbb{T}^{2} \rightarrow \mathbb{T}^{2}$,

$$
a(x, y)=A\left(\begin{array}{l}
x \\
y
\end{array}\right) \quad \bmod 1, \quad \text { where } \quad A=\left(\begin{array}{ll}
2 & 1 \\
1 & 1
\end{array}\right)
$$


More generally, Franks showed that (1) with (2) is semi-conjugate to a [Fra70], i.e., there is a continuous, onto map $k: \mathbb{T}^{2} \rightarrow \mathbb{T}^{2}$ such that

$$
k \circ f=a \circ k .
$$

The map $k$ depends continuously on $g$, and when $g$ is strictly monotone, $k$ is a homeomorphism, implying - as mentioned above - that $f$ is then conjugate $a$.

In [Ler10], the first author made an attempt to elucidate the features of (1) when the circle map $g$ acquires a critical fixed point,

$$
g\left(x_{p}\right)=D g\left(x_{p}\right)=0 .
$$

In this case (1) has a parabolic fixed point $p=\left(x_{p}, 0\right)$ and is no longer Anosov. The main result of [Ler10] was to show that the diffeomorphism acquires elliptic behavior when it crosses this Anosov boundary. Another feature of this map is the separation of the phase space into two regions, one in which the dynamics is nonhyperbolic and the other in which the diffeomorphism appears to be nonuniformly hyperbolic. Though neither of these statements were proved in [Ler10], considerations in favor of these statements were presented.

In this paper we try to use numerical methods to substantiate the following assertions about (1) under the assumptions (2) and (5).

- There is an invariant, open region $E \subset \mathbb{T}^{2}$ whose boundary is formed from the stable and unstable manifolds of two fixed points of the map, a hyperbolic saddle, $h$, and a parabolic point, $p$. The Lebesgue measure of $E$ is strictly less than that of $\mathbb{T}^{2}$.

- The channel $E$ contains all non-hyperbolic orbits of $f$, and indeed has elliptic orbits for generic $\varepsilon>0$.

- Conversely, the dynamics of $\left.f\right|_{H}$, where $H=\mathbb{T}^{2} \backslash E$, is nonuniformly hyperbolic; that is, the map is ergodic in $H$ and has positive Lyapunov exponent.

Of course, these statements are purely numerical observations, which should therefore be considered mathematically as conjectures.

\section{A Parabolic Standard Map}

Following [Lew80, Ler10], we study the dynamics of (1) using the degree-one circle map

$$
g(x)=x+\frac{1}{2 \pi}[\mu-(1+\varepsilon) \sin (2 \pi x)]
$$

where $\mu$ and $\varepsilon \geq 0$, see Fig. 1 . Note that when $\varepsilon=-1$ and $\mu=0$, the map (1) reduces to Arnold's cat map (3).

The map $f$ is a diffeomorphism whenever $g$ is smooth. Indeed

$$
f^{-1}(x, y)=(x-y, y-g(x-y)) .
$$




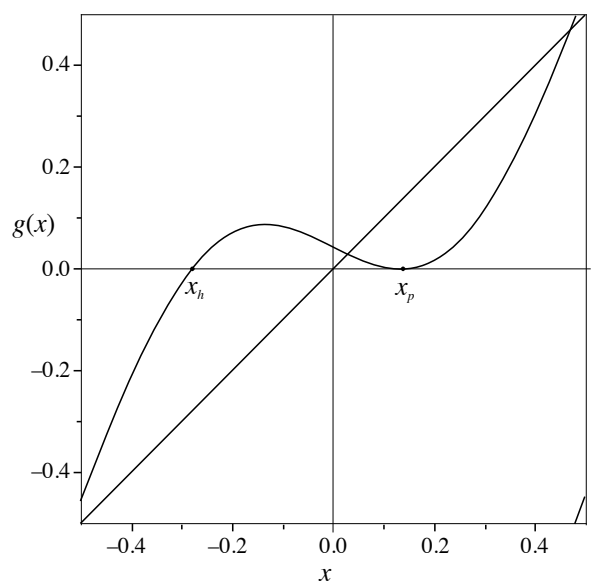

Figure 1: Graph of the force $g(6)$, for $\varepsilon=0.5$ and $\mu=\mu_{p}(\varepsilon) \approx 0.27696$ from (9), with the parabolic point $x_{p} \approx 0.13386$ and saddle $x_{h} \approx-0.27889$.

Moreover, this map is reversible, $f \circ S=S \circ f^{-1}$, with the "second" reversor of Chirikov's map (it does not have the first reversor since $g$ is not odd when $\mu \neq 0$ ),

$$
S(x, y)=(x-y,-y)
$$

with the fixed set $\operatorname{Fix}(S)=\{(s, 0): s \in \mathbb{T}\}$. Note that since $S$ is an involution, the map

$$
f \circ S(x, y)=S \circ f^{-1}(x, y)=(x-2 y+g(x-y),-y+g(x-y))
$$

is also a reversor, with the fixed set

$$
\operatorname{Fix}(f \circ S)=\left\{s+\frac{1}{2} g(s), \frac{1}{2} g(s): s \in \mathbb{T}\right\} .
$$

Under the assumption (5), $g\left(x-x_{p}\right)=\mathcal{O}\left(\left(x-x_{p}\right)^{2}\right)$, the map (1) has a (symmetric) parabolic fixed point $p=\left(x_{p}, 0\right)$. For the case (6) this fixed point occurs at

$$
x_{p}=\frac{1}{2 \pi} \sec ^{-1}(1+\varepsilon)=\frac{1}{\pi} \sqrt{\frac{\varepsilon}{2}}\left(1-\frac{5}{12} \varepsilon+\mathcal{O}\left(\varepsilon^{2}\right)\right),
$$

when $\mu$ is chosen to be

$$
\begin{aligned}
\mu=\mu_{p}(\varepsilon) & \equiv(1+\varepsilon) \sin \left(2 \pi x_{p}\right)-2 \pi x_{p}=\sqrt{\varepsilon(2+\varepsilon)}-\sec ^{-1}(1+\varepsilon) \\
& =\frac{\sqrt{8}}{3} \varepsilon^{3 / 2}\left(1-\frac{9}{20} \varepsilon+\mathcal{O}\left(\varepsilon^{2}\right)\right) .
\end{aligned}
$$

Note that since $D g\left(x_{p}\right)=0$, the Jacobian

$$
D f=\left(\begin{array}{cc}
1+D g(x) & 1 \\
D g(x) & 1
\end{array}\right)
$$


at $x_{p}$ has a double eigenvalue 1 with a nontrivial Jordan block. Moreover, as was shown in [Ler10], the first nonzero coefficient in the nonlinear normal form is quadratic in $x-x_{p}$.

We will fix $\mu=\mu_{p}(\varepsilon)$ using (9) and think of $f$ as a one-parameter family $f_{\varepsilon}$. This family also has a (symmetric) hyperbolic saddle fixed point $h=\left(x_{h}, 0\right)$ where $x_{h}<0$ is the negative root of $g(x)$,

$$
x_{h}=-\frac{\sqrt{2 \varepsilon}}{\pi}\left(1-\frac{19}{60} \varepsilon+\mathcal{O}\left(\varepsilon^{2}\right)\right) .
$$

This is a hyperbolic fixed point of $f$ since $D g\left(x_{h}\right)>0$ whenever $\varepsilon>0$; indeed its multipliers are

$$
\lambda_{ \pm}=1 \pm \sqrt{3 \varepsilon}+\frac{3}{2} \varepsilon+\mathcal{O}\left(\varepsilon^{3 / 2}\right)
$$

The pair of fixed points $\left(x_{p}, 0\right)$ and $\left(x_{h}, 0\right)$ are born from a degenerate saddle at the origin for $\varepsilon=\mu=0$.

Additional fixed points may exist when $\varepsilon$ is large enough so that $g(x)$ is a nonzero integer for some $x \in\left[-\frac{1}{2}, \frac{1}{2}\right]$. For example a saddle-center bifurcation creates a pair of fixed points near $(x, y)=(-0.2151,0)$ when $\varepsilon \approx 3.603$. However, we will study the case that $\varepsilon$ is much smaller than this so that $f$ has only two fixed points.

\section{Invariant Manifolds and the Channel}

The region $E$ mentioned in $\S 1$ is an open domain that is bounded by segments of the stable and unstable manifolds of the parabolic and saddle fixed points $p$ and $h$. In a sense, the construction we follow is similar that of a "DA-map" on the torus [Sma67]. In that case, the unstable manifold of a saddle of an Anosov map is blown-up to make a channel $E$ in such a way that it contains the original unstable fixed point, and its boundaries become unstable manifolds of two saddles. However, since (1) is symplectic we cannot blow up a single manifold; instead, the curve to be blown-up corresponds to the right going halves of the unstable $W^{u}(O)$ and stable $W^{s}(O)$ manifolds of the degenerate saddle $O=(0,0)$ of the almost-hyperbolic diffeomorphism $f_{0}$ at $\varepsilon=\mu=0$. Near the degenerate saddle, these two right-going curves form a cusp-shaped separatrix. Since $f_{0}$ is conjugate to an Anosov map, these manifolds are dense on the torus, and they intersect on a dense set of homoclinic points. Moreover, at every intersection point (except for the degenerate saddle itself), the manifolds cross transversely [Lew80]. As a consequence, if we thicken these two curves we must get a Cantor-like set on the torus. Our simulations support these conclusions, though the situation is more complex. To avoid these complications, we do not attempt this blow-up construction, but instead use a bifurcation technique.

As noted in $\S 2$, when $\varepsilon>0$ the family $f_{\varepsilon}$ has two fixed points: a hyperbolic saddle $h$ and a parabolic point $p$. Each fixed point has smooth, stable, $W^{s}$, and unstable, $W^{u}$, manifolds; numerical approximations are shown in Fig. 2. The existence of the manifolds of the saddle follows from the standard Stable Manifold Theorem [HPS77]. For the parabolic point, existence follows from the results of Fontich [Fon99]. In contrast to the saddle, the curves $W^{s, u}(p)$ are one-sided and they start as a cusp of the form

$$
W^{s, u}(p)=\left\{\left(x, \alpha\left(x-x_{p}\right)^{3 / 2}\right)+\mathcal{O}(\varepsilon): x \geq x_{p}\right\}
$$


with $\alpha=\mp \sqrt{\frac{2 \pi}{3}}[\varepsilon(2+\varepsilon)]^{1 / 4}$, see App. A. As will be discussed in $\S 5$, the manifolds of the parabolic point are asymptotic to the right-going branches of the manifolds of the hyperbolic point; this is seen numerically when the manifolds are extended, as shown in Fig. 3.

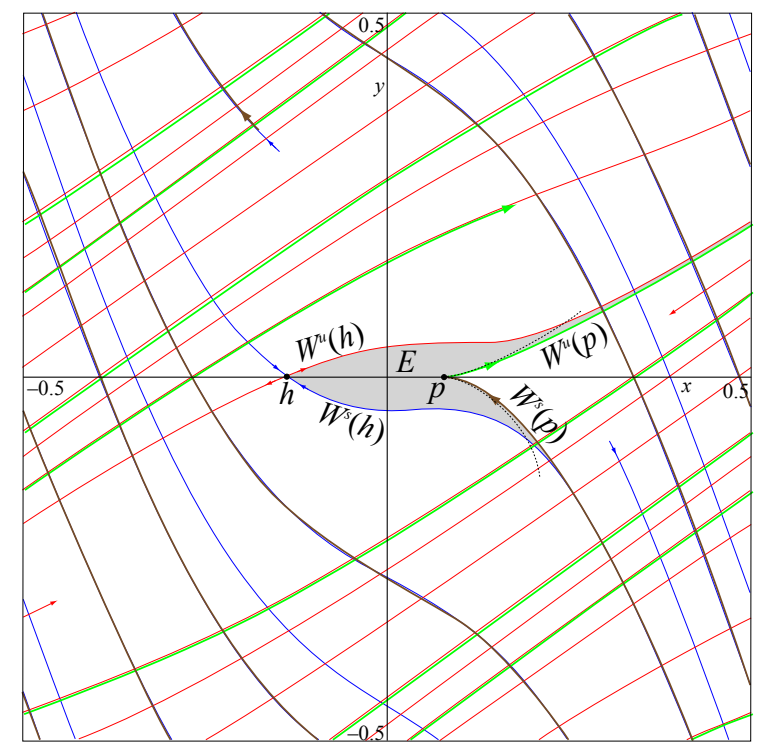

Figure 2: Stable (brown) and unstable manifolds (green) for the parabolic fixed point $p=\left(x_{p}, 0\right)$ and for the saddle $h=\left(x_{h}, 0\right)$ (blue and red, respectively) for the map (1) with $(6), \varepsilon=0.1$, and $\mu=\mu_{p}(\varepsilon) \approx 0.029558$. Also shown (dashed curves) is a $10^{\text {th }}$ degree polynomial approximation (21) for the parabolic manifolds. The gray region is the channel $E$.

Since the right-going unstable manifolds of $p$ and $h$ are born out of the degenerate manifold $W_{0}^{u}$, one can prove that they depend continuously on $\varepsilon$; moreover, any finite segment of these manifolds detached from the fixed point can be proven to vary continuously in the $C^{1}$-topology. In particular, if $T$ is any segment transverse to the local unstable manifold of the degenerate saddle, then the local manifolds $W^{u}(h)$ and $W^{u}(p)$ that emerge will continue to transversely intersect $T$ when $\varepsilon$ is small enough.

Since the map has the reversor $S(7)$, and the points $h$ and $p$ are symmetric, their corresponding stable manifolds are obtained by applying $S$ to the unstable manifolds, and the same continuity and transversality conditions apply.

The initial part of the upper half of the channel, $E^{+}$, has a boundary beginning with the rightgoing manifold $W^{u}(h)$ from $h$ until it first intersects the vertical segment $T=\left\{\left(\frac{1}{2}, y\right): y \in\left[0, \frac{1}{2}\right)\right\}$. For small enough $\varepsilon$, this segment is transverse to both $W^{u}(h)$ and $W^{u}(p)$, so follow $T$ to $T \cap W^{u}(p)$ and then continue along the parabolic manifold back to $p$. Connecting $p$ to $h$ along the $x$-axis closes the boundary of the initial part of $E^{+}$. Similarly, the lower half of the channel, out to the diagonal boundary $S(T)$, is $E^{-}=S\left(E^{+}\right)$. The union is shown in gray in Fig. 2. The remainder of the channel is then obtained by forward and backward iteration.

One has to remark that when $\varepsilon>0$, the behavior of manifolds for $h$ and $p$ is considerably more complex than that for $\varepsilon=0$ - especially because of the formation of folds. This is more easily seen for larger values of $\varepsilon$ : Fig. 4 shows the manifolds for $\varepsilon=0.5$. The size and number of these folds 

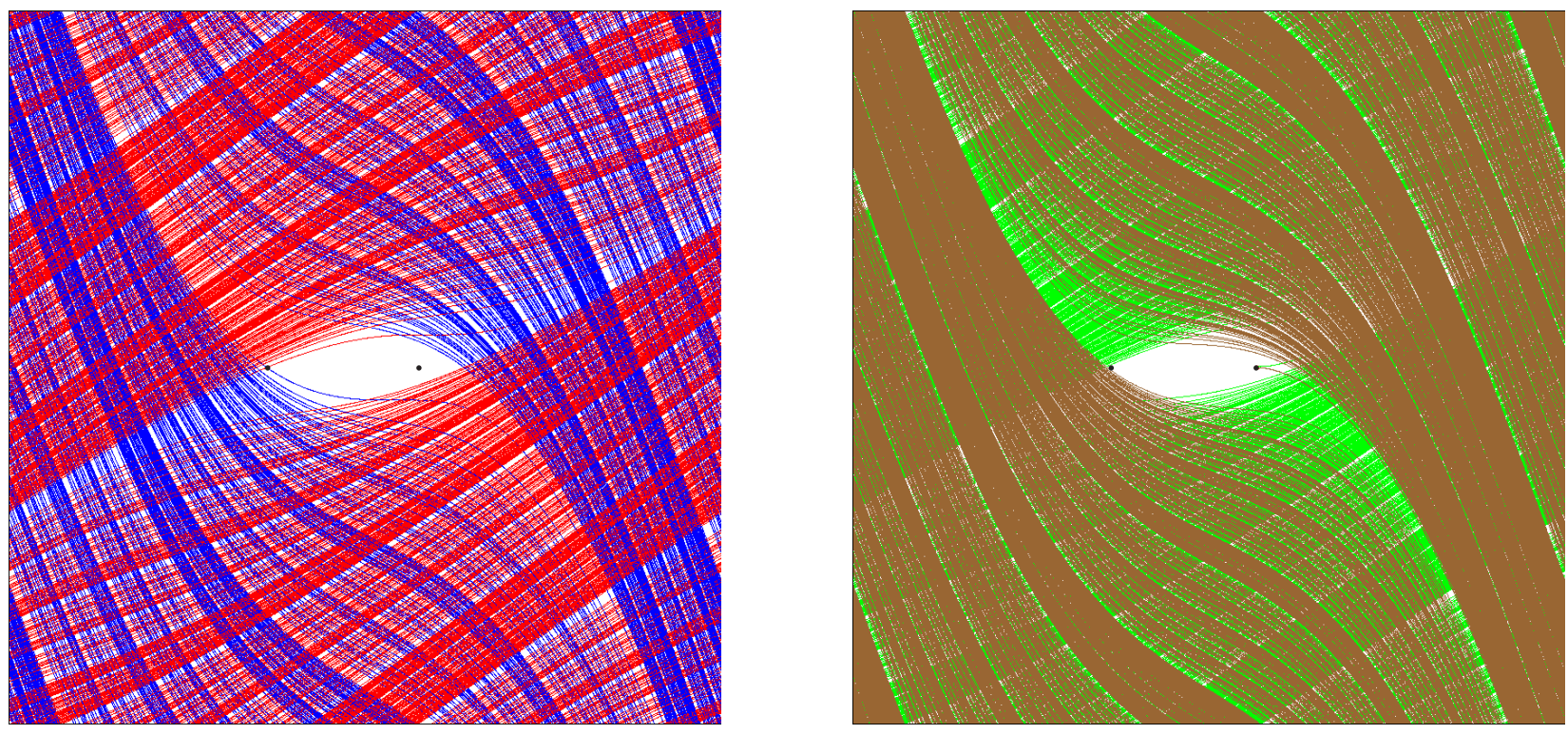

Figure 3: Numerically computed stable and unstable manifolds for the saddle $\left(x_{h}, 0\right)$ (left) and the parabolic point $\left(x_{p}, 0\right)$ (right) for the map (1) with (6), $\varepsilon=0.1$, and $\mu=\mu_{p}(\varepsilon)$. The bounds of the figure are $\left[-\frac{1}{2}, \frac{1}{2}\right]^{2}$.

on any finite segments grows with $\varepsilon$.

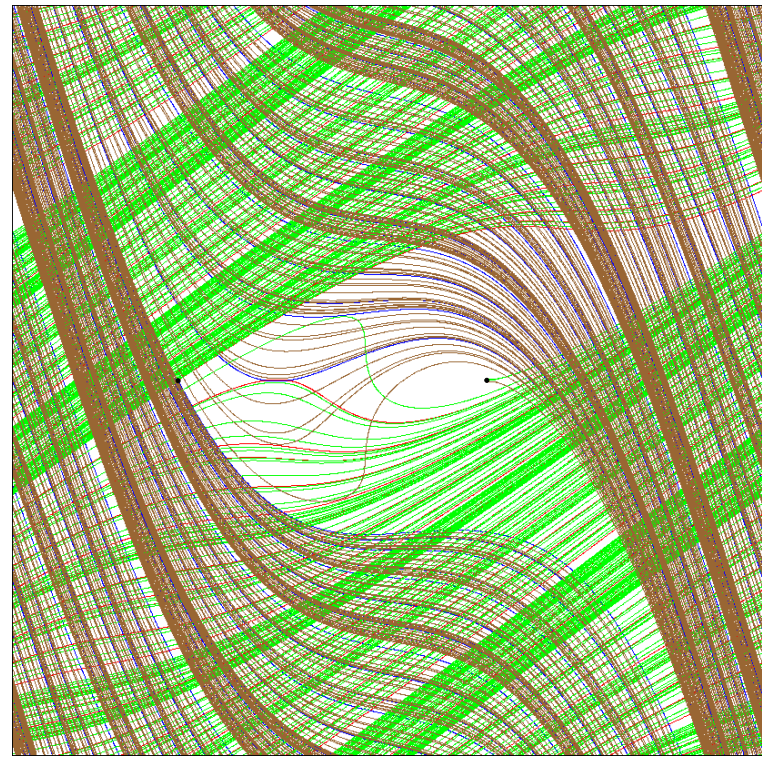

Figure 4: Numerically computed stable and unstable manifolds for the saddle (blue and red) and parabolic (brown and green) fixed points for (1) with (6), $\varepsilon=0.5$, and $\mu=\mu_{p}(\varepsilon)$. The bounds of the figure are $\left[-\frac{1}{2}, \frac{1}{2}\right]^{2}$. The manifolds for the saddle $h$ are mostly obscured because they were plotted before those of $p$.

These folds are caused by homoclinic intersections. To see this, choose a segment of $W^{u}(O)$ 
for the almost-hyperbolic diffeomorphism $f_{0}$ that is long enough to transversely intersect $W^{s}(O)$ at a primary homoclinic point $q_{0}$. Since the perturbed manifolds of $p$ and $h$ are $C^{1}$-close to the degenerate manifolds when $\varepsilon>0$, the unstable manifolds will transversely intersect both of the stable channel boundaries near $q_{0}$. This gives rise to a rectangle $R$, a portion of the channel that is re-injected into itself, see Fig. 5. Two of the corners of $R$ are homoclinic to $p$ and $h$, and two are heteroclinic from/to $p$ and $h$. Since iteration causes points to move monotonically along the stable manifolds, the corners on $W^{s}(h)$ monotonically tend to $h$, and the two on $W^{s}(p)$ monotonically approach $p$. However, the interiors of the sides of the rectangle formed from the unstable manifolds are inside $E$, so they must move along the channel and tend to $W^{u}(h)$ and $W^{u}(p)$. Therefore, these segments lengthen along $W^{u}(h)$, but have endpoints tied to $W^{s}(h)$ and $W^{s}(p)$. This makes them form a fold, like that in Fig. 5 .

For each such fold in the unstable manifolds, there is a corresponding fold in the stable manifolds obtained by applying the reversor (7). Suppose two such symmetric folds intersect. If one decreases $\varepsilon \rightarrow+0$, these two symmetric folds tend to the related points on the unstable and stable separatrices of the degenerate saddle. A consequence is that these two pieces of the stable and unstable manifolds of the saddle have to touch each other for some value of $\varepsilon>0$ on some point of the symmetry line $y=0$. This gives a homoclinic tangency. Near tangencies can be seen in Fig. 4. This story requires a proof, of course, but shows one mechanism for the creation of a tangency.

\section{Elliptic Dynamics}

It is known that the quadratic homoclinic tangencies (as can be seen in Fig. 4) and the resulting Newhouse phenomena [New77, Dua08, GTS07] create elliptic orbits. However, there are no visible elliptic orbits in the numerical computations when $\varepsilon$ is small. As is seen in Fig. 6, when $\varepsilon$ is not too large the map appears numerically to be ergodic in the sense that a single orbit lands in every pixel in a computer generated image. The first visible loss of ergodicity on this scale is due to a periodfive, saddle-center bifurcation at $\varepsilon \approx 1.159$, which creates a chain of five elliptic islands inside the channel. These islands undergo the usual sequence of area-preserving resonant bifurcations, before being destroyed by period-doubling near $\varepsilon=1.274$.

For smaller $\varepsilon$, the islands are too small - in size and/or interval of stability - to be easily observed numerically. Indeed, for $0.01 \leq \varepsilon \leq 1.15$ in steps of $\Delta \varepsilon=0.01$, we observe no islands in the phase space with diameter larger than $10^{-3}$.

Nevertheless, such orbits can be found using the reversibility of the map and thus exploiting its geometric properties. To this end, recall an assertion from the theory of reversible maps [LR98]:

Theorem 1 (Devaney, 1976). Suppose that $\mathcal{O}(z)$ is a symmetric periodic orbit of a reversible diffeomorphism $f$ with a reversing involution $S$ having a smooth submanifold of fixed points $\operatorname{Fix}(S)$. Then:

- $\mathcal{O}(z)$ has period $2 p$ if and only if there exists $\zeta \in \mathcal{O}(z)$ such that $\zeta \in \operatorname{Fix}(S) \cap f^{p}(\operatorname{Fix}(S))$ or $\zeta \in \operatorname{Fix}(f \circ S) \cap f^{p}(\operatorname{Fix}(f \circ S))$;

- $\mathcal{O}(z)$ has period $2 p+1$ if and only if there exists $\zeta \in \mathcal{O}(z)$ such that $\zeta \in \operatorname{Fix}(S) \cap f^{p}(\operatorname{Fix}(f \circ S))$.

Consequently, in order to find a symmetric periodic orbit one should search among the intersections of the images of $\operatorname{Fix}(S)$ and $\operatorname{Fix}(f \circ S)$. In the two-dimensional case, if the intersection of 


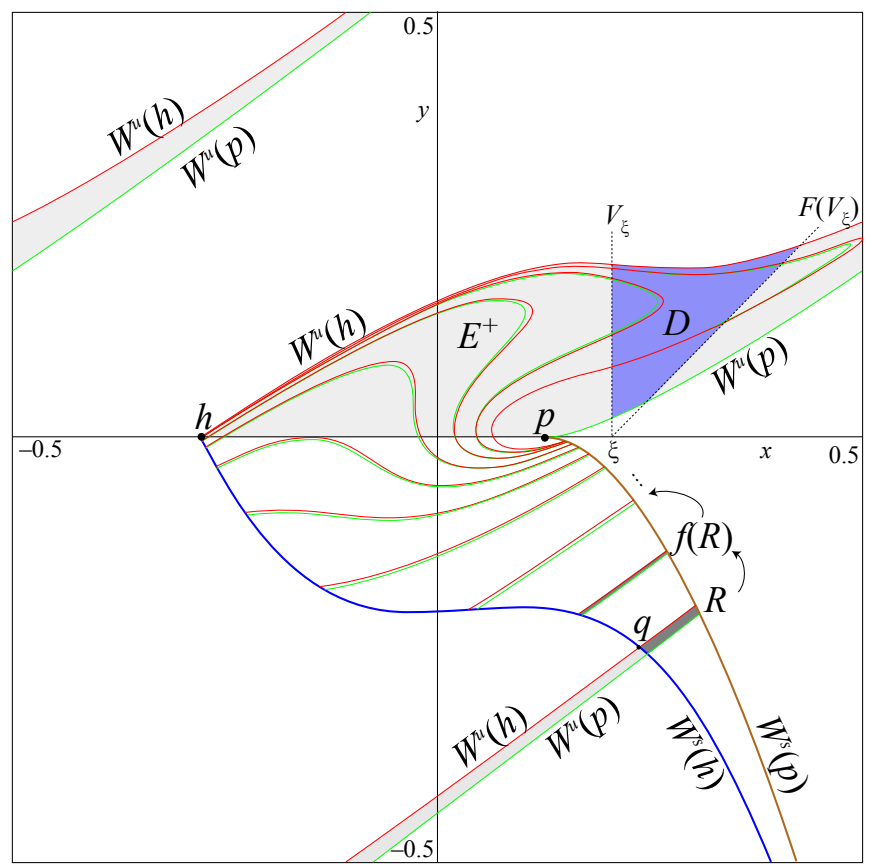

Figure 5: Channel for $\varepsilon=0.5$ and $\mu_{p}(0.5)$. Shown are segments of the stable and unstable manifolds of $h$ and $p$ as in Fig. 2. The unstable channel, $E^{+}$is shaded gray, and a fundamental domain, $D$, is blue. The curves show the first re-injection of the unstable manifolds into the channel and subsequent formation of a fold. The unstable manifolds are clipped when they first cross the stable boundary of the channel $E$. The transversal crossing creates a rectangle $R$ (dark gray) whose corner points are heteroclinic from/to $h$ or $p$. A primary homoclinic point $q$ is labeled. Nine images of $R$ are shown. Upon iteration the unstable sides of the rectangle begin to fold due to their accumulation onto $W^{u}(h)$.

these two sets is transverse then this orbit is a saddle or an elliptic point. If this intersection point is a quadratic tangency, then the orbit is parabolic, and generically corresponds to a saddle-center bifurcation upon varying a parameter. The proof of this statement is in the App. B.

This indeed is confirmed by calculation. ${ }^{1}$ For example, using the reversor (7), we found a pair of period-12 orbits born on $\operatorname{Fix}(S) \cap f^{6}(\operatorname{Fix}(S))$ in a saddle-center bifurcation at $\varepsilon \approx 0.37970034$. For slightly larger $\varepsilon$, one of these orbits is elliptic, and is surrounded by an island, see Fig. 7. As is typical, this island is also surrounded by other elliptic points - a prominent period- $12 \times 7$ orbit is visible - and this orbit also surrounded by more elliptic periodic orbits, for example one of period $12 \times 7 \times 6=504$. This island has stable orbits only for a parameter window of width $\Delta \varepsilon \approx 2(10)^{-7}$.

We conjecture that there are similar, higher-period, elliptic orbits for arbitrarily small, positive $\varepsilon$. However, even a numerical investigation of this seems very difficult.

\footnotetext{
${ }^{1}$ The authors thank A. Kazakov for-upon our request-finding the island in Fig. 7.
} 


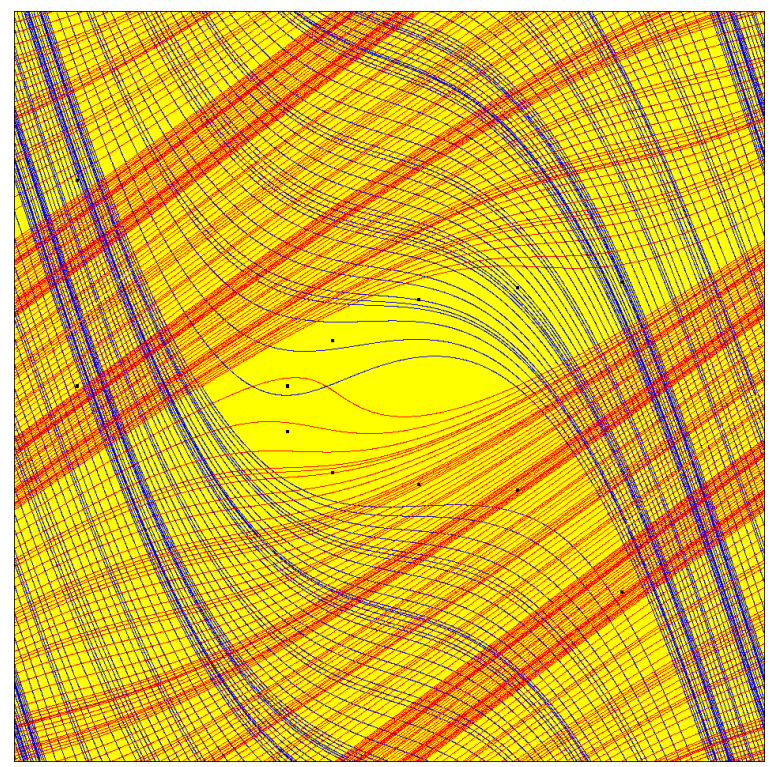

Figure 6: Numerically computed stable (blue) and unstable (red) manifolds the hyperbolic point $h$ of (1) with (6), $\varepsilon=0.37970035$, and $\mu=\mu_{p}(\varepsilon)$. The background (yellow) shows $10^{7}$ iterates of $(0,0)$; these cover every pixel in the $950 \times 950$ pixel image. Nevertheless, there is an elliptic period-12 orbit in the channel; it is covered by the black boxes (the boxes are much larger than the islands!). An enlargement of one of these islands is in Fig. 7.

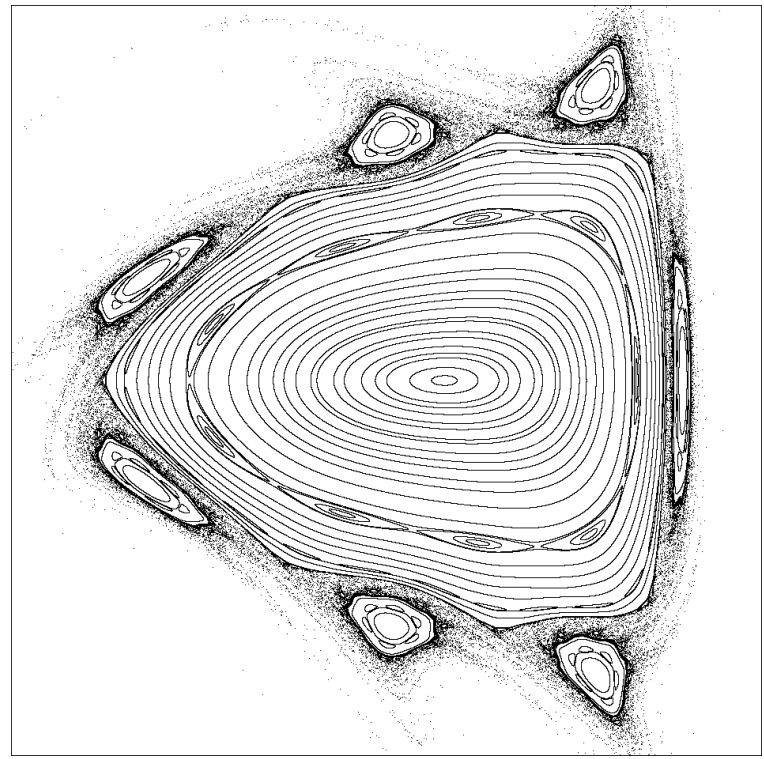

Figure 7: Island around a period-12 elliptic point for (1) with (6) for $\varepsilon=0.37970035$ and $\mu=\mu_{p}(\varepsilon)$. The bounds of this figure are $[-0.13527,-0.13505] \times\left[-5.5(10)^{-8}, 5.5(10)^{-8}\right]$. 


\section{Asymptotic Behavior of the Channel}

Let $F: \mathbb{R}^{2} \rightarrow \mathbb{R}^{2}$, be a lift of $f$, chosen so that its fixed points, which we will call $\tilde{h}$ and $\tilde{p}$, lie in the fundamental square $\left[-\frac{1}{2}, \frac{1}{2}\right) \times\left[-\frac{1}{2}, \frac{1}{2}\right)$. The lifted unstable manifolds, $W^{u}(\tilde{h})$ and $W^{u}(\tilde{p})$, see Fig. 8, are observed to be asymptotic to a ray with fixed slope, and indeed to be asymptotic to one another. Of course, reversibility implies that the same properties hold for the stable manifolds (with another slope).

To explain this, we first parameterize the manifold $W^{u}(\tilde{h})$ in the following standard way. Take some point $(\hat{x}, \hat{y})=\hat{\zeta} \in W^{u}(\tilde{h})$, and let

$$
\ell=W^{u}(\zeta, F(\hat{\zeta}))
$$

be the fundamental segment of unstable manifold connecting $\zeta$ to $F(\zeta)$. By selecting $\zeta$ close enough to $\tilde{h}$ it is always possible to have $\ell$ belong to the unit square. We parameterize $\ell=\{\hat{\zeta}(s): s \in[0,1]\}$ so that $\hat{\zeta}(0)=\hat{\zeta}$ and $\hat{\zeta}(1)=F(\hat{\zeta})$. This parameterization can be extended to $\mathbb{R}$ using iteration so that $s+1$ corresponds to the image.

$$
\hat{\zeta}(s+1)=F(\hat{\zeta}(s))
$$

and $s-1$ to the preimage, etc. Since the iterates of $\ell$ cover $W^{u}(\tilde{h})$, we get a full parameterization:

$$
W^{u}(\tilde{h})=\{\hat{\zeta}(s)=(\hat{x}(s), \hat{y}(s)): s \in \mathbb{R},\}
$$

Note that $\hat{\zeta}(s) \rightarrow \tilde{h}$ as $s \rightarrow-\infty$.

Computing the manifolds up to $x=1000$ and for values $0.01 \leq \varepsilon \leq 1.0$, we observe that

$$
\frac{y_{h}(s)}{x_{h}(s)}=\phi^{-1}+\mathcal{O}\left(\frac{1}{x_{h}(s)}\right)
$$

where $\phi=\frac{1}{2}(1+\sqrt{5})$ is the golden mean. The same considerations apply to the unstable manifold of the parabolic point $W^{u}(\tilde{p})$. To verify these observations, we first recall that the map (1) is semi-conjugate to the linear, Anosov map $a$, (3), i.e., that there is a continuous, onto map $k$ that is homotopic to the identity such that (4) holds [Ler10]. In particular, this implies that $f$ induces a map $f^{*}=a^{*}$ on the fundamental group $\mathbb{Z}^{2}$ of the torus that is hyperbolic. The Anosov map $a$ has a unique fixed point at the origin, a saddle with eigenvalues $\phi^{2}$ and $\phi^{-2}$. The right-going unstable manifold of the origin, is the projection of the right-going unstable eigenvector of the matrix $A$ onto the torus. This has slope $\phi^{-1}$-and of course this slope is precisely the slope that we observe in (13).

We will prove the following result.

Theorem 2. Let $F$ be a lift of (1) to the plane and assume that the force $g$ is of the form (6) with $\varepsilon$ small enough. Then the right-going unstable manifolds of the saddle $\tilde{h}=\left(x_{h}, y_{h}\right)$ and parabolic $\tilde{p}=\left(x_{p}, y_{p}\right)$ fixed points of $F$ both tend to $\infty$ and have a limiting slope $\phi^{-1}$, the inverse of the golden mean.

As a start to the proof of Th. 2, we first note that the semi-conjugacy collapses the stable and unstable manifolds of both $p$ and $h$ onto the corresponding manifolds of the fixed point of the Anosov map: 


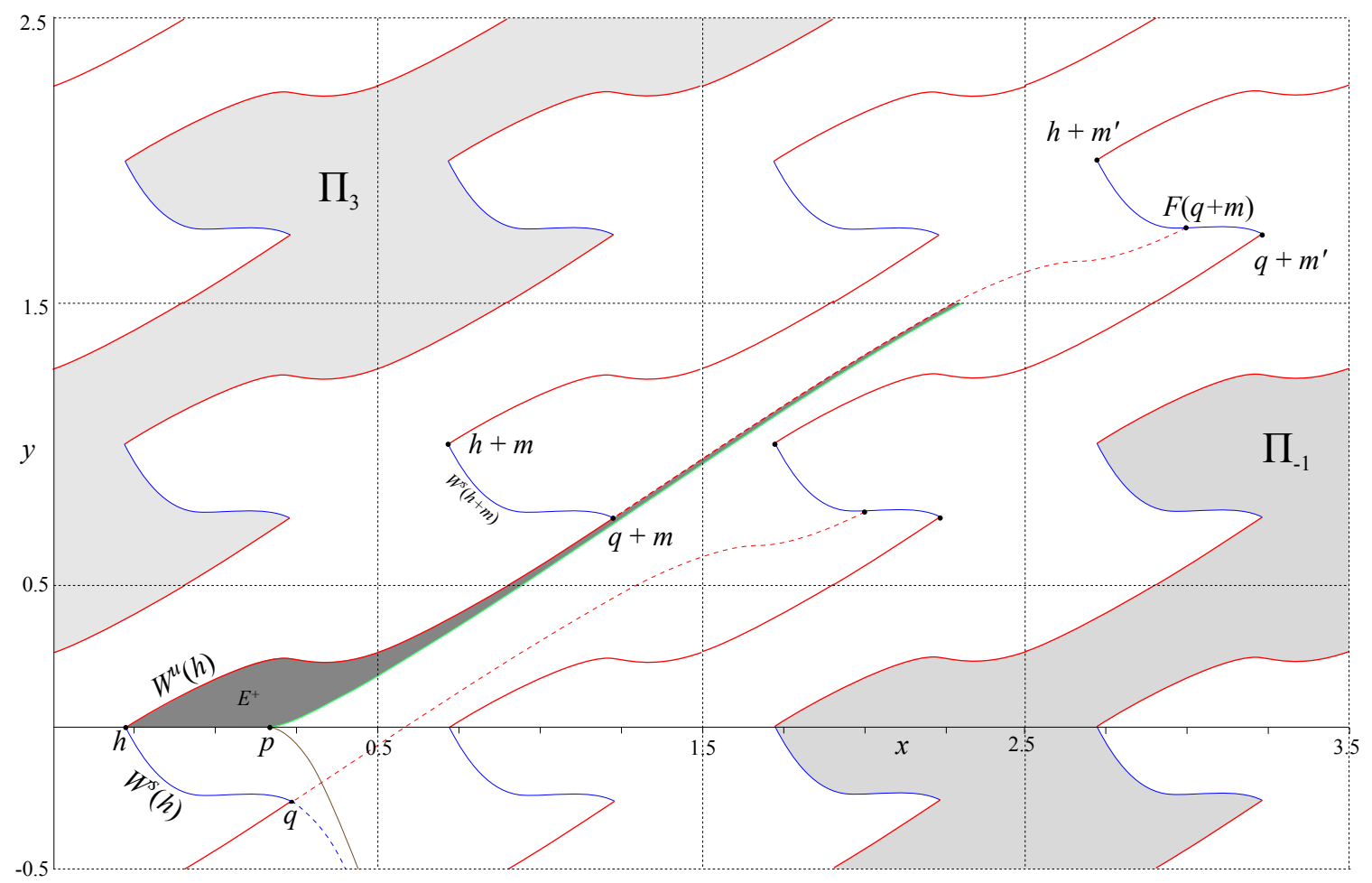

Figure 8: Stable (blue) and unstable (red) manifolds of the hyperbolic fixed point, and stable (brown) and unstable (green) manifolds of the parabolic fixed point for $\varepsilon=0.6$ for the lift $F$. The dark gray region, labeled $E^{+}$, is a portion of the upper half of the lifted channel. Also shown are the strips constructed from the lifts of manifold segments $W^{u, s}(h, q)$ to a homoclinic point $q$; two of these, labeled $\Pi_{3}$ and $\Pi_{-1}$, are shaded light gray. The image $F(q+m)$ lies on the stable segment $W^{u}\left(h+m^{\prime}, q+m^{\prime}\right)$ where $m^{\prime}=(3,2)$.

Lemma 3. The semi-conjugacy $k$ transforms $W^{u}(h)$, the upper boundary of the channel $E$ for $f$, onto the unstable manifold $\gamma=W^{u}(O)$ of $O=(0,0)$ under the Anosov map a. The same is true for $W^{u}(p)$. Similarly both $W^{s}(h)$ and $W^{s}(p)$ are mapped by $k$ onto the stable manifold of $O$ under $a$.

Proof. The map $f$ has exactly two fixed points: $h$, and $p$, and the map $a$ has the unique fixed point, $O$. Using (4), the $k$-image of a fixed point for $f$ must be a fixed point for $a$, and hence $k(p)=$ $k(h)=O$. By definition, the backward orbit of any point $\zeta \in W^{u}(h)$ tends to $h: \zeta_{t}=f^{t}(\zeta) \rightarrow h$ as $t \rightarrow-\infty$. Under the semi-conjugacy, one has $k\left(\zeta_{t}\right)=a^{t} \circ k(\zeta)$, thus the $k$-image of the backward orbit of $\zeta$ is the backward orbit of the point $k(\zeta)$. Since $k$ is continuous, $k\left(\zeta_{t}\right) \rightarrow k(h)=O$ as $t \rightarrow-\infty$, thus $k(\zeta) \in \gamma$. Thus $k\left(W^{u}(h)\right)$ is a subset of $\gamma$. To prove that this image is onto $\gamma$ we need to find a fundamental segment in $W^{u}(h)$ whose image is a fundamental segment in $\gamma$. One way to do this is to recall that for $\varepsilon=0$ the map $k_{0}$ is a homeomorphism. Therefore since $k_{\varepsilon}$ depends continuously on $\varepsilon$, when $\varepsilon$ is small enough, the $k$-images of the distinct points $\zeta, f_{\varepsilon}(\zeta)$ are distinct. Thus the image of the fundamental segment in $W^{u}(h)$ covers a fundamental segment of $\gamma$.

By a similar argument, the same results hold for the stable manifold. 
The main tool we will use in the proof of Th. 2 is a theorem stated by Weil in 1935 at the Moscow Topological Conference [Wei36], but proved much later by Markley [Mar69]. Let $L$ be a continuous, semi-infinite, simple (without self-intersections) curve on the torus $\mathbb{T}^{2}=\mathbb{R}^{2} / \mathbb{Z}^{2}$ and $\tilde{L}=\left\{(x(t), y(t)): t \in \mathbb{R}^{+}\right\}$be its parameterized lift to the plane. Let $\rho(x, y)$ denote the standard Euclidean distance from $(x, y)$ to the origin. Then the following theorem holds.

Theorem 4 (Weil (1935)). If $\rho(x(t), y(t))$ tends to infinity as $t \rightarrow \infty$, then $\tilde{L}$ has an asymptotic direction; that is, either there exists a slope $m^{*} \in \mathbb{R}$ such that $\lim _{t \rightarrow \infty} y(t) / x(t)=m^{*}$, or, if this ratio is unbounded, then $\lim _{t \rightarrow \infty} x(t) / y(t)=0$.

In other words, if the lifted curve $(x(t), y(t)), t \in[0, \infty)$, has no finite accumulation points (i.e., there is no sequence $t_{n} \rightarrow \infty$ such that $\left.\lim _{n \rightarrow \infty} \rho\left(x\left(t_{n}\right), y\left(t_{n}\right)\right)=\left(x_{*}, y_{*}\right)\right)$, then it has an asymptotic direction.

Given these results, we now proceed to prove our theorem:

Proof of Th. 2. Choose lifts $F$ and $K$ of the maps $f$ and $k$ to the plane such that the fixed points $\tilde{h}$ and $\tilde{p}$ of $F$ lie in the unit square $\left[-\frac{1}{2}, \frac{1}{2}\right) \times\left[-\frac{1}{2}, \frac{1}{2}\right)$ and that $K(\tilde{h})=K(\tilde{p})=O$. We are to prove that the unstable manifolds $W^{u}(\tilde{h})$ and $W^{u}(\tilde{p})$ of $F$ go to infinity and have an asymptotic direction equal to $\phi^{-1}$ - the same as that of the unstable direction of the linear map $A$. For the first part, we will apply Th. 4, and thus we only need to verify that both of the lifted curves have no finite accumulation points. We shall prove this for $W^{u}(\tilde{h})$, since the proof for $W^{u}(\tilde{p})$ is the same.

Begin by choosing a primary, transverse homoclinic point $q \in W^{u}(h) \cap W^{s}(h),{ }^{2}$ and consider the segments $W^{u}(h, q)$ and $W^{s}(h, q)$, with orientations from $h$ to $q$. We claim that it is possible to choose $q$ so that $W^{s}(h, q)$ lies in the interior of the unit square. Consequently, the forward images, $f^{t}(q)$, remain in a neighborhood of $h$, moving monotonically from $q$ to $h$ along the local segment $W^{s}(h, q)$. Note that the tangent vectors at $q$ to $W^{u}(h)$ and $W^{s}(h)$ form a frame. Since $f$ is symplectic and thus orientation-preserving, the orientation of this frame is preserved under $D f$.

The existence of such a $q=q_{\varepsilon}$ follows from the $C^{1}$ closeness of the manifolds of $f_{\varepsilon}$ to those of $f_{0}$, apart from an $\mathcal{O}(\sqrt{\varepsilon})$-neighborhood of $O$, and the existence of a transverse homoclinic point on the right-going unstable manifold of $W^{u}(O)$ for $f_{0}$. Indeed [Lew80] showed that the intersections of the stable and unstable manifolds of $f_{0}$ are transverse everywhere except at $O$. Choose one such primary intersection, $r \in W^{u}(O) \cap W^{s}(O)$. Since $f_{0}^{t}(r) \rightarrow O$ as $t \rightarrow \infty$, there is an image $q_{0}=f^{k}(r)$, such its forward images lie in a ball of radius, say, $\frac{1}{4}$ of $O$. This homoclinic point $q_{0}$ is of course still primary and transverse. Now we take $\varepsilon$ small enough in order that: (1) $q_{0}$ is not in an $\mathcal{O}(\sqrt{\varepsilon})$-neighborhood of $O ;(2)$ the intersection point $q_{0}$ continues to a point $q_{\varepsilon}$ still in the unit square; and (3) the intersection at $q_{\varepsilon}$ remains transverse. This can be done since since the manifolds of $f_{\varepsilon}$ are $C^{1}$ close to those of $f_{0}$.

The loop $\mathcal{C}=\bar{W}^{u}(h, q) \cup \bar{W}^{s}(h, q)$ is a simple (since $q$ is primary) closed curve on the torus. This curve is not homotopic to zero and has some nontrivial representation $\left(m_{1}, m_{2}\right) \in \mathbb{Z}^{2}$, in the fundamental group of the torus. This follows from that fact that $\mathcal{C}$ is homotopic to the loop made up from pieces of $W^{u}(O)$ and $W^{s}(O)$ of $f_{0}$, and hence it is homeomorphic to the related loop of the Anosov map $a$, which is not homotopic to zero.

\footnotetext{
${ }^{2}$ Recall that a homoclinic point $q$ of a plane diffeomorphism with a saddle fixed point $h$ is "primary," if the closed loop $\bar{W}^{u}(h, q) \cup \bar{W}^{s}(h, q)$ has no self-intersection points.
} 
A lift of $\mathcal{C}$ to the covering plane unwinds to an infinite curve that tends to infinity with rational slope $m_{2} / m_{1}$. The collection of all lifts of $\mathcal{C}$ cut the plane into infinite number of disjoint strips, recall Fig. 8. Consider the segment $\mathcal{U}=W^{u}(\tilde{h}, \tilde{q}+m)$ that belongs to the upper boundary of one of these strips, say $\Pi_{1}$. The image $F(\mathcal{U})=W^{u}(\tilde{h}, F(\tilde{q}+m))$ expands and, by orientation preservation, enters the interior of $\Pi_{1}$. The second endpoint, $F(\tilde{q}+m)=F(\tilde{q})+m^{\prime}$, where $m^{\prime}=A m$, lies in the interior of the segment $W^{s}\left(\tilde{h}+m^{\prime}, \tilde{q}+m^{\prime}\right)$, and is not on the upper boundary of $\Pi_{1}$, since $m^{\prime}$ is not parallel to $m$.

Two cases are possible. The first occurs when $F(\mathcal{U})-\mathcal{U}$ intersects the boundary of $\Pi_{1}$ only at $F(\tilde{q}+m)$, i.e., it intersects no other lift of $W^{s}(h, q)$. The implication is that at the next iteration, $F^{2}(\mathcal{U})$ will cross the strip below $\Pi_{1}$, due to preservation of orientation, etc. In this case, the rightgoing manifold $W^{u}(\tilde{h})$ goes to infinity and cannot have accumulation points in finite part of the plane. Similar considerations were used in [Gri77]. However, it may be the case that $F(\mathcal{U})-\mathcal{U}$ intersects some additional lifts of the segment $W^{s}(h, q)$ that belong to the boundary of $\Pi_{1}$. In this second case, $F(\mathcal{U})$ has to leave and then return to $\Pi_{1}$ since its extreme point $F(\tilde{q}+m)$ still exits $\Pi_{1}$ through $W^{s}\left(\tilde{h}+m^{\prime}, \tilde{q}+m^{\prime}\right)$. This leads to a potential problem exemplified by the folds shown in Fig. 5: there could be loops homotopic to zero made up from pieces of $W^{u}(h)$ and $W^{s}(h)$.

Nevertheless, as we show in App. C, using specific properties of the map (1), all points of $W^{u}(\tilde{h})$ tend to infinity. To apply the considerations of App. C, we need to choose a fundamental segment $\ell \in W^{u}(\tilde{h})$ in the first quadrant, such that for all $(x, y) \in \ell$,

$$
x+\phi^{-1} y>\frac{1}{2 \pi}(1+2 \varepsilon) .
$$

To this end, it is enough to verify that a segment $\ell_{0}$ exists for $f_{0}$ (for $\varepsilon=\mu=0$ ), a fact that is easily numerically verified. Then, $\ell_{\varepsilon}$ satisfies the requirement for $f_{\varepsilon}$ for small enough $\varepsilon$, since the unstable manifolds of both $\tilde{h}$ and $\tilde{p}$ are $C^{1}$-close to those of $f_{0}$ on compact sets away from an $\mathcal{O}(\sqrt{\varepsilon})$-neighborhood of $O$ (in fact, $C^{0}$-closeness of manifolds is sufficient).

Therefore we have shown that there are no accumulation points, and Th. 4 applies, and thus $W^{u}(\tilde{h})$ has a limiting slope. The same argument applies to $W^{u}(\tilde{p})$, since it too is mapped onto $\Gamma$ by $K$.

Finally we need to prove that the limiting slope is indeed equal to $\phi^{-1}$. To that end we use (1) with the assumption that $g$ has degree one:

$$
g(x)=x+\hat{g}(x)
$$

where $\hat{g}(x)$ is a continuous, periodic function. If $(\hat{x}(s), \hat{y}(s))=\hat{\zeta}(s)$, then under the map $F(x, y)=$ $\hat{\zeta}(s+1)$. Define the slope of the chord from the fixed point to $\hat{\zeta}(s)$ by

$$
m(s)=\frac{\hat{y}(s)-y_{h}}{\hat{x}(s)-x_{h}} .
$$

Subtracting the fixed point from both sides of (1), and computing the slope gives, after some algebra,

$$
m(s+1)=\frac{1+m(s)+\frac{\hat{g}\left(x_{h}(s)\right)-\hat{g}\left(x_{h}\right)}{x_{h}(s)-x_{h}}}{2+m(s)+\frac{\hat{g}\left(x_{h}(s)\right)-\hat{g}\left(x_{h}\right)}{x_{h}(s)-x_{h}}} .
$$


Now, according to Th. 4 , the slope $m(s)$ has a limit, $m^{*}$. Moreover, since $x_{h}(s)$ is unbounded and $\hat{g}(x)$ is periodic,

$$
\lim _{s \rightarrow \infty} \frac{\hat{g}(\hat{x}(s))-\hat{g}\left(x_{h}\right)}{\hat{x}(s)-x_{h}}=0 .
$$

Thus after taking the limit on both sides of (15) we come to

$$
m^{*}=\frac{1+m^{*}}{2+m^{*}}
$$

which implies, since $m(s)>0$, that $m^{*}=\phi^{-1}$.

Not only do $W^{u}(\tilde{h})$ and $W^{u}(\tilde{p})$ for the lift $F$ have the same limiting slope, as implied by Th. 2, but they converge to each other. This can be seen numerically by choosing the first parameter value for which each curve crosses a particular abscissa value $\xi$. Let $\eta_{h}(\xi)$ and $\eta_{p}(\xi)$ denote the corresponding ordinates on $W^{u}(h)$ and $W^{u}(p)$, respectively. We observe (again computing up to $\xi=1000)$ that the vertical distance between these curves decreases algebraically as

$$
\eta_{h}(\xi)-\eta_{p}(\xi)=\mathcal{O}\left(\xi^{-1}\right)
$$

see Fig. 9. A proof of this result appears to be nontrivial. Indeed, this decay is not uniform - since we define $\xi$ to be the first horizontal crossing, the formation of folds causes the vertical distance to exhibit jumps. More generally, close approaches to the stable channel cause oscillations; the first place this occurs is near $\xi=2.5$. In this case the vertical distance decreases monotonically up to $\xi=2$, it subsequently increases as the unstable channel crosses the initial segment of the stable channel, reaching a local maximum near $\left(x_{h}, y_{h}\right) \approx(2.59,1.64)$. The next two local maxima occur at $(5.39,3.38)$ and $(7.23,4.57)$, again correlated with crossing the stable channel. As can be seen in Fig. 9, these local maxima occur at approximately the same values of $\xi$ for any value of $\varepsilon$. Indeed, the function $\left[\eta_{h}(\xi)-\eta_{p}(\xi)\right] \xi$ appears to be quasiperiodic, with two dominant periods $\Delta \xi=2.61$ and 6.90, again independent of $\varepsilon$.

\section{Channel Area}

Our goal in this section is to compute the area contained in the channel $E$ "between" the invariant manifolds of the parabolic and hyperbolic points, and to show that, when $\varepsilon$ is small, the total area of the channel is less than one, implying that the dynamics of $f$ is partitioned into two invariant regions of nonzero measure. As noted above, and in particular in Fig. 6, it is numerically infeasible to simply iterate chosen initial conditions in the channel, since these numerical trajectories rapidly fill every pixel in an image. Thus, instead, we will use numerical computations of the stable and unstable manifolds that form the boundaries of $E$.

For the lift $F$, the region "between" the curves $W^{u}(\tilde{h})$ and $W^{u}(\tilde{p})$ corresponds to the upper, unstable half, $\tilde{E}^{+}$, of the lifted channel, e.g., the dark gray region in Fig. 8. Its area can be easily computed up to some finite extension $\xi$ on the plane. Let $\tilde{E}^{+}(\xi) \subset \mathbb{R}^{2}$ denote the region with boundary

$$
\partial \tilde{E}^{+}(\xi)=\left\{(x, 0): x_{h} \leq x \leq x_{p}\right\}+W^{u}\left(\tilde{p}, \zeta_{p}\right)+V_{\xi}-W^{u}\left(\tilde{h}, \zeta_{h}\right)
$$




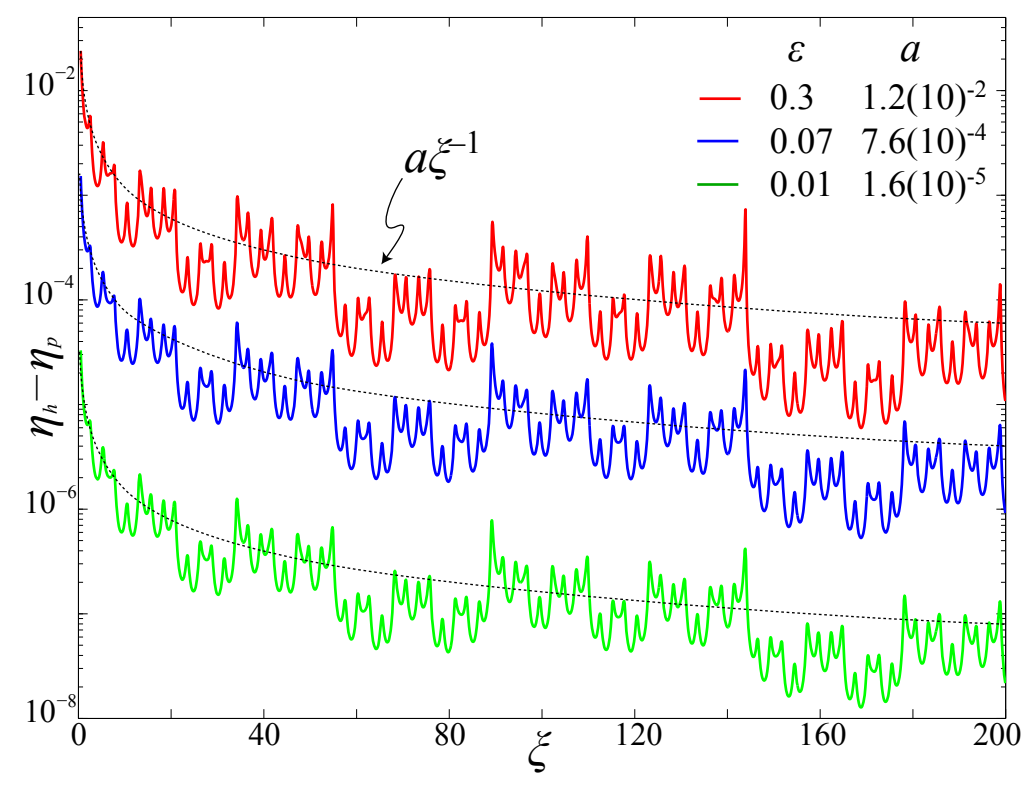

Figure 9: Vertical distance between the unstable manifolds of the saddle and parabolic fixed points for the lift of (1) up to $\xi=200$ for three values of $\varepsilon$. Also shown (dashed curves) are the graphs $a / \xi$, with the values of $a$ as shown.

where $\zeta_{h, p}=\left(\xi, \eta_{h, p}(\xi)\right)$ are points on the unstable manifolds, and

$$
V_{\xi}=\left\{(\xi, y): \eta_{p}(\xi) \leq y \leq \eta_{h}(\xi)\right\}
$$

is the connecting vertical segment. Let

$$
\tilde{A}^{+}(\xi)=\operatorname{Area}\left(\tilde{E}^{+}(\xi)\right)
$$

denote the area of the unstable channel up to $\xi$. This can be most easily computed using the relation between action and area, see App. D. The results are shown in Fig. 10 for several values of the cut-off $\xi$, as a function of $\varepsilon$.

Since, by (8) and (11), $x_{p}-x_{h}=\mathcal{O}\left(\varepsilon^{1 / 2}\right)$, and the slope of the unstable eigenvector of (10) at $x_{h}$ is $\mathcal{O}\left(\varepsilon^{1 / 2}\right)$, it can be seen that $\tilde{A}^{+}(\xi)=\mathcal{O}\left(\varepsilon^{3 / 2}\right)$. More precisely, this follows from the Hamiltonian normal form valid near $\varepsilon=\mu=0$. This asymptotics is supported by the calculations shown in Fig. 10.

Note that $\tilde{A}_{\varepsilon}^{+}(\xi)$ must be unbounded as $\xi \rightarrow \infty$ : this is a consequence of area preservation. Indeed, the unstable channel can be generated by iteration of a "fundamental domain." For each point $\zeta=(\xi, \eta)$, on a branch of an unstable manifold, the segment $\ell=W^{u}(\zeta, F(\zeta))$ generates the entire manifold under iteration. A fundamental domain, $D$, for the unstable half of the channel corresponds to the region with boundary

$$
\partial D=W^{u}\left(\zeta_{p}, F\left(\zeta_{p}\right)\right)+F\left(V_{\xi}\right)-W^{u}\left(\zeta_{h}, F\left(\zeta_{h}\right)\right)-V_{\xi},
$$

where $\zeta_{h, p}=\left(\xi, \eta_{h, p}\right)$ are points on the respective manifolds, recall Fig. 5 . Note that the image $F\left(V_{\xi}\right)$ of the vertical segment (18) is a line segment with unit slope since (1) has constant twist. 


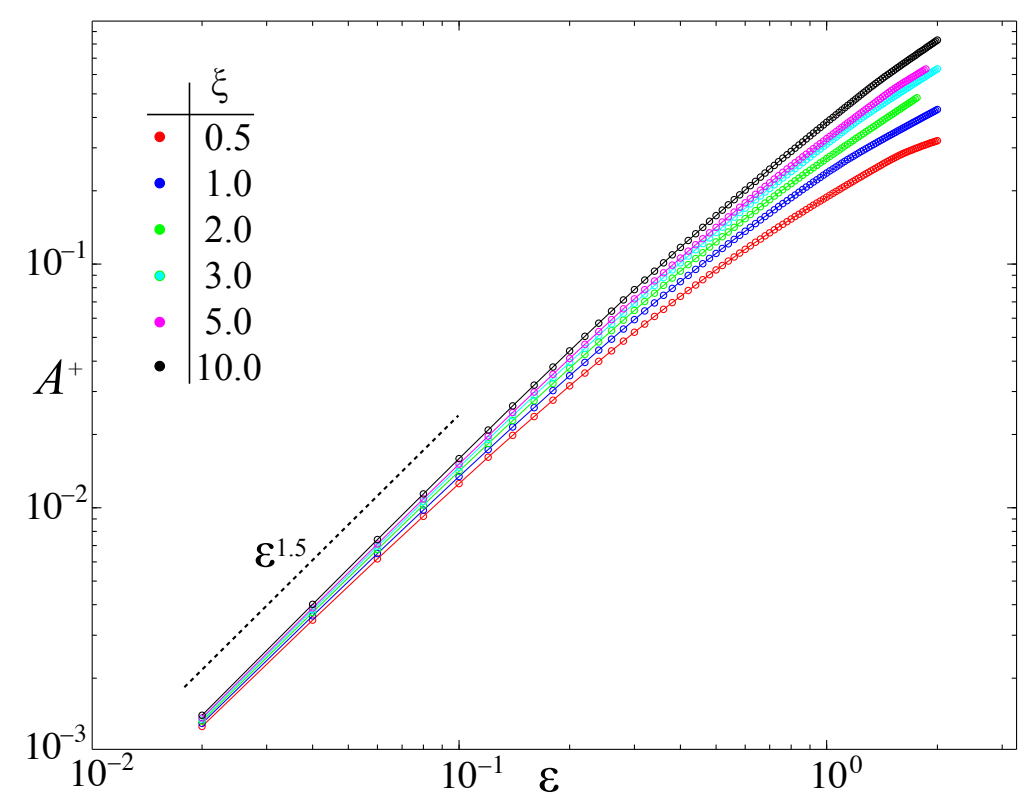

Figure 10: Area of the lift of the upper, unstable half of the channel for the lift $F$ of (1) as a function of $\varepsilon$ for six values of the cut-off $\xi$.

The channel $\tilde{E}^{+}$is generated by the images of $D$, and thus, with each iteration of the map, its area grows by the area of the fundamental domain.

Since $\tilde{A}^{+}(\xi) \rightarrow \infty$ as $\xi \rightarrow \infty$, the vertical distance between the manifolds, (16), cannot decrease more rapidly than $\xi^{-1}$, confirming the decay observed in Fig. 9. Given this rate of convergence, it is clear that $A^{+}$must increase logarithmically with the intercept $\xi$, and this is confirmed by the computations in Fig. 11. The oscillations seen in this figure correspond to those seen in the vertical distance in Fig. 9.

Since the lifted channel has infinite area, its projection onto $\mathbb{T}^{2}$ necessarily has self- intersections. These correspond to the re-injection of the rectangular pieces of the channel into the region near the origin, as we noted in $\S 3$, and showed in Fig. 5. Forward images of $R$ intersect $E^{+}$and these areas are to be deleted from the lifted area of the channel upon projection.

To compute the area of $E=E^{+} \cup E^{-}$on $\mathbb{T}^{2}$ accounting for the overlap of the channel with itself, we resort to an image-based calculation. To start, the region $\tilde{E}^{+}$is computed up to an extension $\xi$ as before. This region is projected into $\mathbb{T}^{2}$, and discretized into an $N \times N$ pixel image - a pixel is deemed to be occupied if there is a point on the manifolds $W^{u}(h)$ or $W^{u}(p)$ that lands in the pixel. The region is computed by filling the pixels vertically from $\eta_{p}$ to $\eta_{h}$. We fill in the channel sequentially, increasing the cut-off $\xi$; in this case the folds, which are in the interior of the channel, do not cause a problem with the filling algorithm. Finally the full channel is computed by applying the reflection $S$ to the pixels in the unstable channel, giving the image $E_{N \times N}$. An example, for $\varepsilon=0.5$, is shown in Fig. 12 .

We observe that as the number of pixels, $N$, grows, the computed channel area monotonically decreases, and that the error is proportional to $N^{-1}$, see Table 1. We can use this to extrapolate to get an estimate of the area to an absolute error less than $10^{-4}$, the column labeled $b_{N}$ in the 


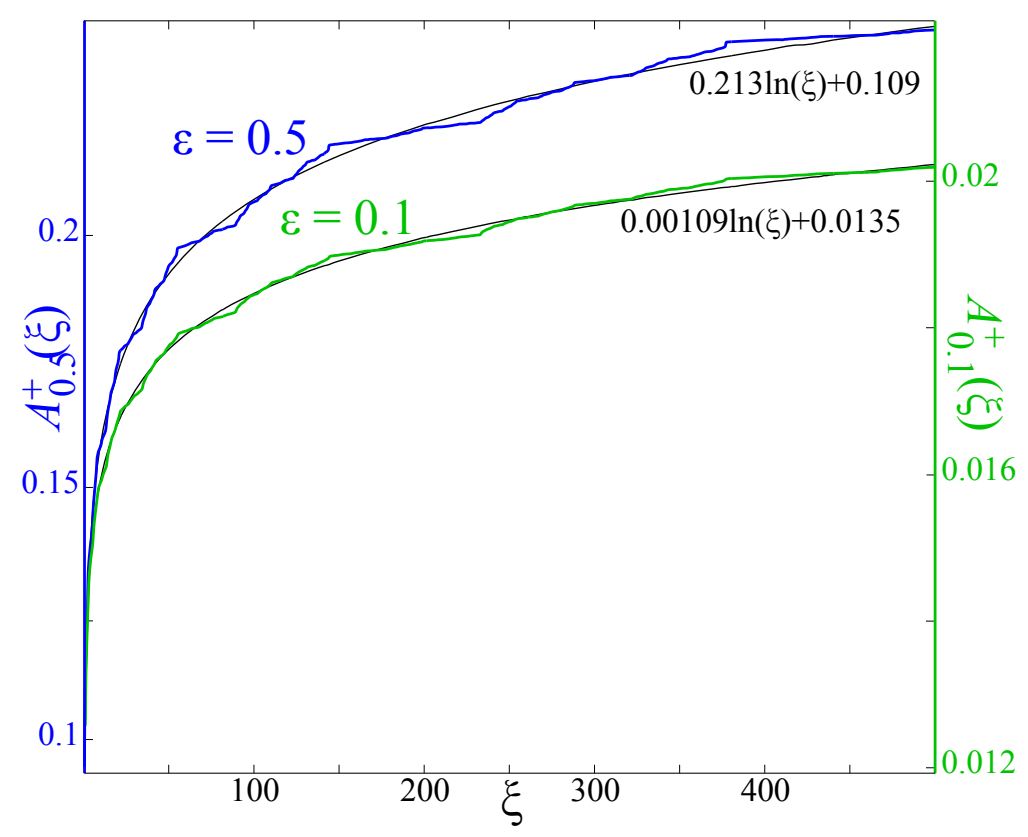

Figure 11: Area $A^{+}$of the upper channel on the plane as a function of the horizontal extent, $\xi$ of the channel for $\varepsilon=0.5$ (blue, left axis) and $\varepsilon=0.1$ (green, right axis). Fits (black) are to the log functions shown using data up to $\xi=1000$.

table. A final extrapolation to remove errors proportional to $N^{-2}$, the column $c_{N}$, reduces the error estimate slightly. Thus we estimate that

$$
A_{0.1}(100)=0.03679 \pm 2(10)^{-5}
$$

Note that the area of the upper lifted channel (computed using the action) is $\tilde{A}_{0.1}^{+}(100)=0.018563$, which when doubled gives a total channel area of 0.037126 . Thus the fraction of area excluded due to overlap is about $0.9 \%$.

After this extrapolation, we vary the cut-off, $\xi$, to attempt to estimate $A_{\varepsilon}=\lim _{\xi \rightarrow \infty} \operatorname{Area}\left(E_{\varepsilon}(\xi)\right)$. The results, again for $\varepsilon=0.1$ are shown in Table 2. After the second extrapolation, we estimate that the true area of the channel is

$$
A_{0.1}=0.03990 \pm 5(10)^{-5}
$$

Using these ideas, we compute the area as a function of $\varepsilon$ for three values of the channel cut-off, $\xi$, see Fig. 13. For $\varepsilon<0.01$, the results have not converged: they depend on $\xi$ significantly. It is strange that for these values, $A(200)>A(300)$; this is due to error in the extrapolations for $b_{N}-$ none of the computed values $a_{N}(\xi)$ have this contradictory property. The error bars in the figure are estimated by $\left|b_{32000}-b_{16000}\right|$. When $\varepsilon>0.01$, the area seems to have converged with $\xi=300$. As in Fig. 10 the area again grows as $\varepsilon^{3 / 2}$ when $\varepsilon$ is small. A fit over the interval $0.01<\varepsilon<1.0$ gives

$$
A_{\varepsilon}=(1.02 \pm 0.09) \varepsilon^{1.42 \pm 0.07},
$$




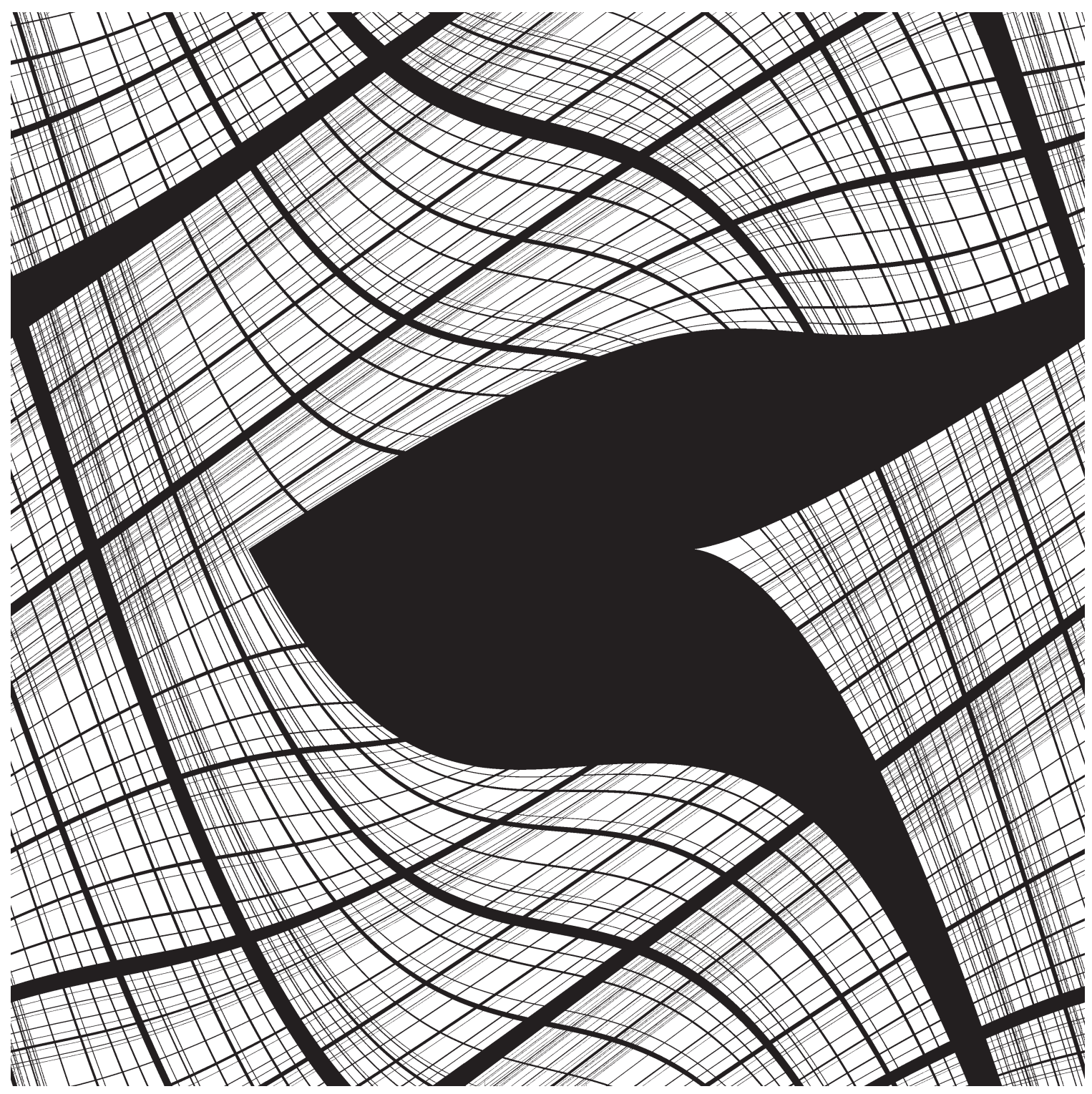

Figure 12: Discretized channel $E_{N \times N}$ for $\varepsilon=0.5$ with $\xi=100$ and $N=4000$. The channel (black region) intersects $7,040,240$ pixels, or $44.00 \%$ of the area. For $\xi=100, \eta_{h}-\eta_{p}=1.47(10)^{-4}$, so that the channel height is less then one pixel. Note that the folds in the manifolds are hidden since they occur in the interior of the channel, recall Fig. 4.

while a fit over the narrower interval $0.04<\varepsilon<0.6$ gives an exponent of $1.49 \pm 0.04$. When $\varepsilon$ approaches 1, the power law predicts that $A_{\varepsilon} \rightarrow 1$, and, as can be seen in the figure, the area saturates near the value one. 


\begin{tabular}{r|ccc}
\multicolumn{1}{c|}{$N$} & $a_{N}$ & $b_{N}$ & $c_{N}$ \\
\hline 500 & 0.486252 & & \\
1000 & 0.289216 & 0.092180 & \\
2000 & 0.167454 & 0.045692 & 0.030196 \\
4000 & 0.103127 & 0.038800 & 0.036503 \\
8000 & 0.070165 & 0.037203 & 0.036671 \\
16000 & 0.053519 & 0.036873 & 0.036763 \\
32000 & 0.045162 & 0.036805 & 0.036782 \\
64000 & 0.040973 & 0.036784 & 0.036777 \\
128000 & 0.038890 & 0.036807 & 0.036815 \\
\hline
\end{tabular}

Table 1: Area of the discretized channel, $a_{N}$, for $\varepsilon=0.1$ and $\xi=100$ as a function of the number of pixels, $N \times N$, in the image. The extrapolation, $b_{N}=2 a_{N}-a_{N / 2}$, removes errors $\mathcal{O}\left(N^{-1}\right)$ and the second, $c_{N}=\frac{1}{3}\left(4 b_{N}-b_{N / 2}\right)$, removes errors $\mathcal{O}\left(N^{-2}\right)$.

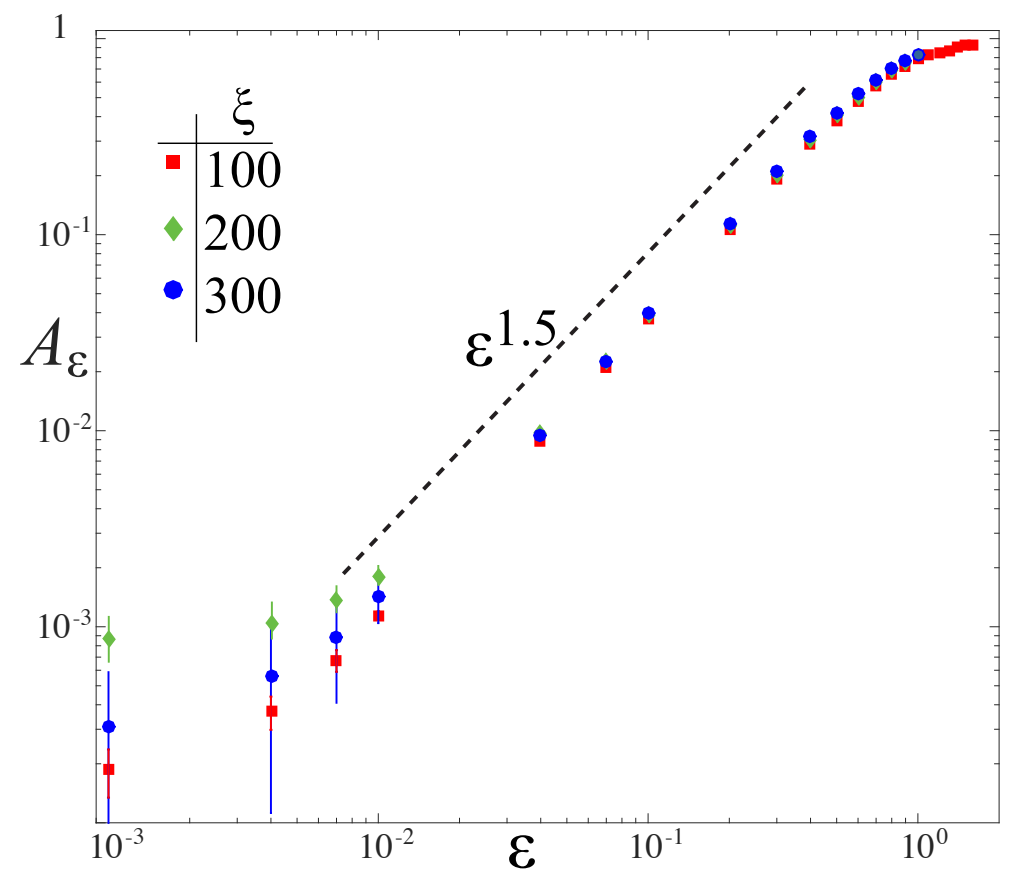

Figure 13: Area of the channel $E$ using the second order extrapolation as in Table 1, for $N=32,000$, as a function of $\varepsilon$ for three values of the channel cut-off, $\xi$, as shown. Error, estimated as the difference $\left|b_{32000}-b_{16000}\right|$, is only visible when $\varepsilon \leq 0.01$

\section{Lyapunov Exponents}

The Lyapunov exponents of the family $f_{\varepsilon}$ appear, by the standard computation, to be positive. We compute the finite-time exponent

$$
\lambda_{\varepsilon}(x, y, T)=\frac{1}{T} \ln \left\|D f_{\varepsilon}^{T}(x, y) v_{0}\right\|, \quad v_{0}=\left(\begin{array}{l}
0 \\
1
\end{array}\right)
$$




\begin{tabular}{r|ccc|cc|c}
$\xi$ & $a_{8000}$ & $a_{16000}$ & $a_{32000}$ & $b_{16000}$ & $b_{32000}$ & $c_{32000}$ \\
\hline 30 & 0.043929 & 0.039000 & 0.036549 & 0.034071 & 0.034098 & 0.034107 \\
70 & 0.059595 & 0.047852 & 0.041964 & 0.036109 & 0.036076 & 0.036065 \\
110 & 0.073580 & 0.055382 & 0.046242 & 0.037184 & 0.037102 & 0.037075 \\
150 & 0.087464 & 0.062759 & 0.050329 & 0.038054 & 0.037899 & 0.037847 \\
190 & 0.102100 & 0.070515 & 0.054570 & 0.038930 & 0.038625 & 0.038523 \\
230 & 0.116212 & 0.077799 & 0.058356 & 0.039386 & 0.038913 & 0.038755 \\
270 & 0.128380 & 0.084259 & 0.061867 & 0.040138 & 0.039475 & 0.039254 \\
310 & 0.141428 & 0.091035 & 0.065402 & 0.040642 & 0.039769 & 0.039478 \\
350 & 0.154256 & 0.097727 & 0.068905 & 0.041198 & 0.040083 & 0.039711 \\
390 & 0.167162 & 0.104657 & 0.072596 & 0.042152 & 0.040535 & 0.039996 \\
430 & 0.180157 & 0.112024 & 0.076516 & 0.043891 & 0.041008 & 0.040047 \\
470 & 0.192669 & 0.119685 & 0.080630 & 0.046701 & 0.041575 & 0.039866 \\
510 & 0.205416 & 0.126197 & 0.083939 & 0.046978 & 0.041681 & 0.039915
\end{tabular}

Table 2: Channel area for $\varepsilon=0.1$ as a function of the cut-off $\xi$. Columns labeled $a_{N}$ are the computed areas for $N \times N$ pixels. The final three columns show extrapolations $b_{N}=2 a_{N}-a_{N / 2}$, and $c_{N}=\frac{1}{3}\left(4 b_{N}-b_{N / 2}\right)$.

for an initial condition $(x, y)$ with the vertical initial deviation vector $v_{0}$ over a time $T$. The results shown in Fig. 14 give the mean exponent for 400 initial conditions with $T=10^{4}$ (for these parameters standard deviation of the distribution of exponents is smaller than 0.005). Note that $\left\langle\lambda_{0}\right\rangle \approx 0.902177<\ln \phi^{2} \approx 0.9642$, the exponent of the Anosov map (3) (i.e., $\varepsilon=-1$ and $\mu=0$ ). The exponent decreases monotonically from its value at $\varepsilon=0$ until $\varepsilon=1.55$, when it begins to increase (though not monotonically), reaching $\langle\lambda\rangle \approx 1.1$ at $\varepsilon=5$.

To estimate the exponent separately for orbits that lie in the channel $E$ and orbits that lie in its exterior, $H=\mathbb{T}^{2} \backslash E$, we use the $N \times N$-pixel approximation of the channel, $E_{N \times N}(\xi)$, recall Fig. 12 . Initial conditions for the exterior computation are chosen in each pixel of $H_{N \times N}(\xi)=\mathbb{T}^{2} \backslash E_{N \times N}(\xi)$, and each is iterated only over the time that it remains in $H_{N \times N}(\xi)$ : the time $T$ in (20) is chosen so that the orbit segment from 0 to $T$ lies in $H$. Similarly, an in-channel, Lyapunov exponent can be computed by choosing initial conditions in $E_{N \times N}(\xi)$, iterating them only as long as they remain in the approximate channel. The resulting finite-time Lyapunov exponents are shown, for $\varepsilon=0.1$, as a function of their initial condition in Fig. 15 using a channel cut-off of $\xi=50$. The mean exponent for initial conditions in $H$ is $\left\langle\lambda_{0.1}\right\rangle_{H}=0.8352$, while $\left\langle\lambda_{0.1}\right\rangle_{E}=0.5123$.

The mean exponents in $H$ and $E$ are also shown in Fig. 14 as a function of $\varepsilon$. Since these computations are for a fixed number of pixels, $N=3000$, the approximation $H_{N \times N}$ will vanish for large enough $\varepsilon$. Since orbits leave this gridded approximation rapidly, we do not show these curves for $\varepsilon>1$.6. Note that the exponent for initial conditions in $H$ is a monotonically decreasing function of $\varepsilon$, while that for $E$ primarily increases. It appears that the minimum of the globally averaged exponent (dashed curve in the figure) corresponds to the point at which the channel area reaches $\mathcal{O}(1)$ so that essentially all orbits are in the channel. In principle, the globally averaged exponent should be the weighted average of the channel and exterior results - but this is not true for the figure. The reason is that the computations are carried out over different time intervals. The latter two are averages over the shorter time during which orbit segments remain in $E$ or in 


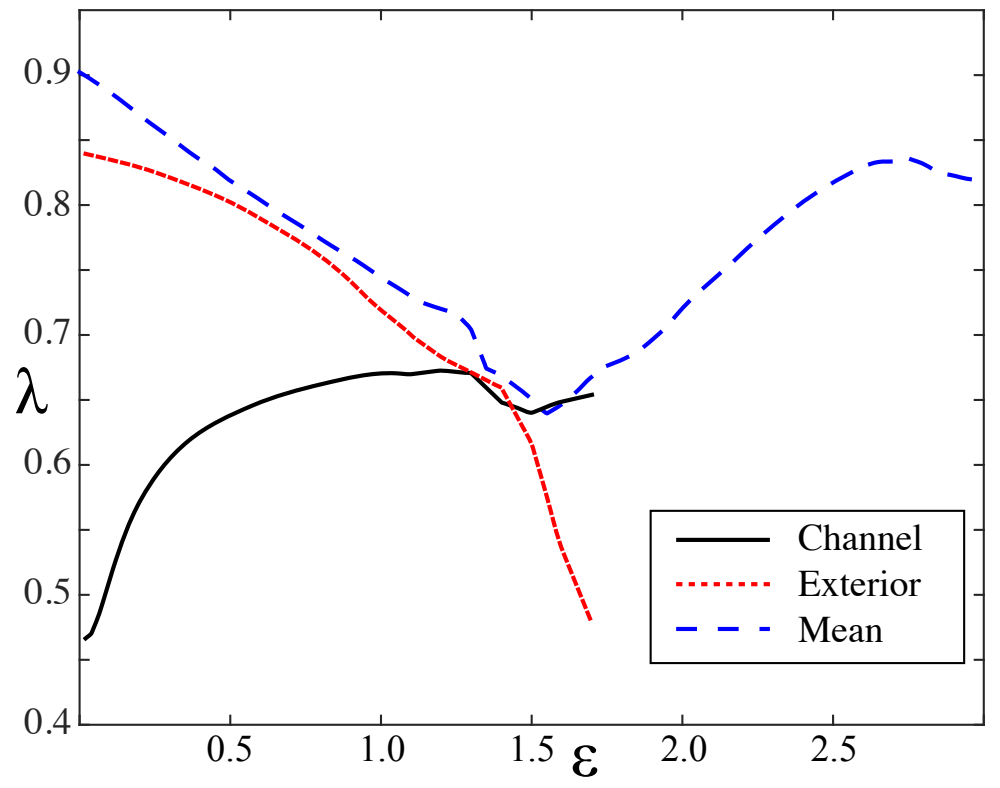

Figure 14: Lyapunov exponents for the map (1) with (6) as a function of $\varepsilon$. The dashed curve (blue) is the average exponent for a grid of $20 \times 20$ initial conditions, each iterated $10^{4}$ steps. The dotted (red) and solid (black) curves show separately the mean exponents for orbits in the exterior and interior of the channel, estimated using $3000 \times 3000$ pixel image with a cut-off $\xi=50$.

$H$. We observe that the value of (20) increases with $T$; the result is that both $\left\langle\lambda_{0.1}\right\rangle_{E}$ and $\left\langle\lambda_{0.1}\right\rangle_{H}$ are smaller than those of the global average, which used $T=10^{4}$.

\section{Conclusions}

We have provided numerical evidence for the three conjectures of $\S 1$ for a family of parabolic standard maps $f_{\varepsilon}: \mathbb{T}^{2} \rightarrow \mathbb{T}^{2}$, (1) with force (6), that are homotopic to the Anosov map (3), but which have a pair of fixed points for each $\varepsilon>0$, one hyperbolic and one parabolic. We showed that the right-going stable and unstable manifolds of these fixed points bound a channel $E \subset \mathbb{T}^{2}$. The lift $\tilde{E}$ of the channel to the plane has unstable boundaries that are asymptotic to lines of slope $\phi^{-1}$, the slope of the unstable manifolds of the Anosov map. Since these maps are, in addition, reversible, the same assertion concerning the slope is valid for stable manifolds. The height of the lifted channel approaches zero as $x^{-1}$, which is the maximal rate consistent with area-preservation.

- We have computed the area $A_{\varepsilon}(E)$ for the lift using the action, and on the torus using pixelbased computations. We show that $A_{\varepsilon}(E)<1$ when $\varepsilon<1$. We conjecture that there is a transition near $\varepsilon=1$ where the measure of the channel reaches one.

- We have found elliptic periodic orbits in the channel for several values of $\varepsilon$. These are formed through saddle-center bifurcations near tangencies of the stable and unstable manifolds of the hyperbolic point, i.e., by the Newhouse mechanism. We conjecture that there are elliptic 


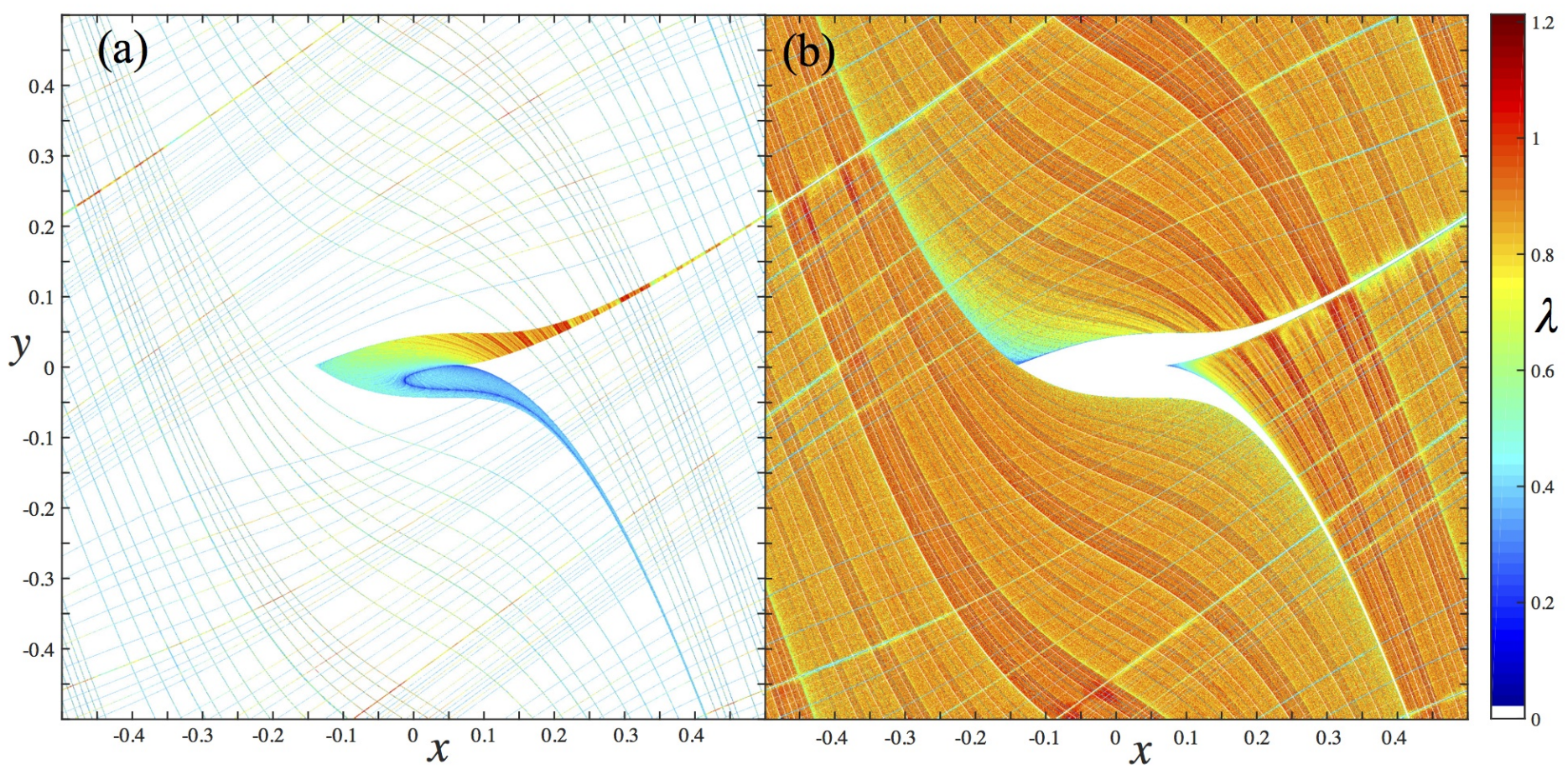

Figure 15: Distribution of the finite-time Lyapunov exponents (20) for $\varepsilon=0.1$. Panel (a) shows initial conditions in the channel and (b) in the exterior of the channel. The channel is computed up to the cut-off $\xi=50$, and discretized onto a $3000 \times 3000$ grid.

orbits in the channel for arbitrarily small, positive $\varepsilon$, and that there are no elliptic orbits in its complement, $H$.

- We have computed finite-time Lyapunov exponents for orbit segments both in the channel $E$ and in its complement, $H$. As $\varepsilon \rightarrow 0^{+}$it appears that the former monotonically decrease, while the latter limit to the exponent of the almost-hyperbolic map $f_{0}$. This occurs even though a naive numerical iteration of any given initial condition appears to fill every pixel of a computed image.

Though our example is not generic since it has a parabolic fixed point, we hope that these results are convincing arguments in favor of the hypothesis that a generic, sufficiently smooth symplectic diffeomorphism does have a positive measure invariant set with positive Lyapunov exponent so that it is non-uniformly hyperbolic on this set. This would show the drastic difference between properties of sufficiently smooth and $C^{1}$-smooth symplectic diffeomorphisms which generically have zero Lyapunov exponent almost everywhere with respect to the Lebesgue measure [Boc02]. 


\section{Appendices}

\section{A Parabolic Manifolds}

As shown by [Fon99], a map of the form (1), with a parabolic fixed point at $\left(x_{p}, 0\right)$ such that $g\left(x-x_{p}\right)=\mathcal{O}\left(\left(x-x_{p}\right)^{2}\right)$ has a pair of stable and unstable manifolds. In the neighborhood of the fixed point, these can be parametrically represented as

$$
W^{u}(p)=\left(\begin{array}{c}
x_{p} \\
0
\end{array}\right)+\left(\begin{array}{c}
s^{2} \\
\alpha_{3} s^{3}+\alpha_{4} s^{4}+\alpha_{5} s^{5}+\ldots
\end{array}\right)
$$

under the assumption that the dynamics on the manifold is parameterized by the one-dimensional $\operatorname{map} \sigma: \mathbb{R} \rightarrow \mathbb{R}$

$$
s \mapsto \sigma(s)=s+\beta_{2} s^{2}+\beta_{3} s^{3}+\ldots
$$

Demanding that this set be invariant gives a set of equations that can be solved, order-by-order, for the coefficients $\left\{\alpha_{i}, \beta_{j}\right\}$. For the case (6), the result is

$$
\begin{array}{rlrl}
\alpha_{3} & =\sqrt{\frac{2 \pi k}{3}}, & \alpha_{4}=-\frac{\pi k}{2}, & \alpha_{5}=\sqrt{\frac{6 \pi^{3}}{k}} \frac{12+11 k^{2}}{144}, \\
\beta_{2}=\sqrt{\frac{\pi k}{6}}, & \beta_{3}=\frac{\pi k}{6}, & \beta_{4}=\sqrt{\frac{6 \pi^{3}}{k}} \frac{4+k^{2}}{96},
\end{array}
$$

where $k \equiv \sqrt{\varepsilon(2+\varepsilon)}$. These expansions are well-defined only for $k \neq 0$, requiring $\varepsilon \neq 0$. Note that since $\alpha_{3}>0, W^{s, u}(p)$ has the form of a cubic cusp.

This expansion, while useful for small $s$, does not give a good representation too far from the fixed point. For example, the degree-10 polynomial approximations are compared with the numerically generated manifolds of $\left(x_{p}, 0\right)$ in Fig. 2 .

\section{B Creation of elliptic points from tangency of fixed point sets}

In this appendix we present a justification of the method of finding elliptic points used in $\S 4$. We consider only the case of an $S$-reversible area-preserving map, $f \circ S=S \circ f^{-1}$ for which the involution $S$ has a smooth line of fixed points, $\operatorname{Fix}(S)$.

Theorem 5. Suppose that $f$ is a $C^{2}$ area-preserving diffeomorphism that is reversible w.r.t. a smooth involution $S$, and the set $\operatorname{Fix}(S)$ of the involution fixed points is a smooth curve. Then if $\xi=\operatorname{Fix}(S) \cap f^{p}(\operatorname{Fix}(S))$ is a point of transversal intersection, it is a point on either an elliptic or a hyperbolic period-2p orbit, while if $\xi$ is a point of quadratic tangency, it is a parabolic period- $2 p$ orbit.

Proof. Since $\xi \in \operatorname{Fix}(S) \cap f^{p}(\operatorname{Fix}(S))$, then $\xi=S(\xi)$ and there is a point $\eta \in \operatorname{Fix}(S)$ such that $f^{p}(\eta)=\xi$. Consider first the case $p=1$. Then

$$
f^{2}(\eta)=f(f(\eta))=f(\xi)=f(S(\xi))=S\left(f^{-1}(\xi)\right)=S(\eta)=\eta .
$$


Similarly, one has $f^{2}(\xi)=\xi$. By induction, the same is true for any $p \in \mathbb{Z}$. Below we work with $p=1$ to facilitate calculations.

According to the Bochner-Montgomery theorem [BM46] we can take two symplectic charts: $\mathcal{V}$ near $\eta$ with coordinates $(x, y)$ and $\mathcal{U}$ near $\xi$ with coordinates $(u, v)$ such that $\left.S\right|_{\mathcal{V}}(x, y)=(x,-y)$, and similarly $\left.S\right|_{\mathcal{U}}(u, v)=(u,-v)$. Moreover, $\left.f\right|_{\mathcal{V}}=f_{1}: \mathcal{V} \rightarrow \mathcal{U}$ is written as follows (we assume with no loss of generality that $\xi$ and $\eta$ have zero coordinates in the related charts)

$$
\left(\begin{array}{l}
u \\
v
\end{array}\right)=A\left(\begin{array}{l}
x \\
y
\end{array}\right)+\left(\begin{array}{c}
F_{1}(x, y) \\
G_{1}(x, y)
\end{array}\right)
$$

where $A$ is a constant matrix and $F_{1}$ and $G_{1}$ are $\mathcal{O}(2)$. Similarly $\left.f\right|_{\mathcal{U}}=f_{2}: \mathcal{U} \rightarrow \mathcal{V}$ has the form

$$
\left(\begin{array}{l}
x \\
y
\end{array}\right)=B\left(\begin{array}{l}
u \\
v
\end{array}\right)+\left(\begin{array}{l}
F_{2}(u, v) \\
G_{2}(u, v)
\end{array}\right)
$$

Note that in both cases, $d u \wedge d v=d x \wedge d y$ by area preservation.

If $\xi$ is the point of transversal intersection of $f_{1}(\operatorname{Fix}(S))$ and $\operatorname{Fix}(S)$, then the vectors $\left(a_{11}, a_{21}\right)^{\top}$ and $(1,0)^{\top}$ are transverse, i.e., $a_{21} \neq 0$. In this case, when $-1<a_{12} a_{21}<0$, the point $\eta$ is elliptic (its eigenvalues satisfy $\left|\lambda_{1,2}\right|=1$ ), while if $a_{12} a_{21}>0$ it is an orientable saddle, and if $a_{12} a_{21}<-1$ it is a non-orientable saddle.

The tangency of $D f_{1}(\operatorname{Fix}(S))$ and $\operatorname{Fix}(S)$ at $\xi$ implies $a_{21}=0$ and area preservation gives $a_{22}=a_{11}^{-1}$. Using reversibility in both coordinate charts implies that

$$
f_{1}^{-1}(u, v)=S \circ f_{2} \circ S(u, v)=\left(\begin{array}{cc}
b_{11} & -b_{12} \\
-b_{21} & b_{22}
\end{array}\right)\left(\begin{array}{l}
u \\
v
\end{array}\right)+\left(\begin{array}{c}
F_{2}(u,-v) \\
-G_{2}(u,-v)
\end{array}\right),
$$

and

$$
f_{2}^{-1}(x, y)=S \circ f_{1} \circ S(x, y)=\left(\begin{array}{cc}
a_{11} & -a_{12} \\
0 & a_{22}
\end{array}\right)\left(\begin{array}{l}
x \\
y
\end{array}\right)+\left(\begin{array}{c}
F_{1}(x,-y) \\
-G_{1}(x,-y)
\end{array}\right) .
$$

Comparing these with the inverses of (23) and (24) implies that:

$$
B=\left(\begin{array}{cc}
a_{22} & a_{12} \\
0 & a_{11}
\end{array}\right)
$$

and $U_{1}(u, v)=F_{2}(u,-v), V_{1}(u, v)=-G_{2}(u,-v), U_{2}(x, y)=F_{1}(x,-y), V_{2}(x, y)=-G_{1}(x,-y)$, where $U_{1}, V_{1}, U_{2}, V_{2}$ are the nonlinear terms of the inverse maps $f_{1}^{-1}, f_{2}^{-1}$. Below, we denote $a_{11}=a_{22}^{-1}=\alpha$ and $a_{12}=\beta$, for brevity.

The quadratic tangency of $f_{1}(\operatorname{Fix}(S))$ and $\operatorname{Fix}(S)$ at $\xi$ implies $\partial^{2} G_{1} / \partial x^{2} \neq 0$ at $(0,0)$. The map $f^{2}$ near a period-two point $\eta$ has the form $f_{2} \circ f_{1}$. Hence, the linear part of this map has the matrix

$$
B A=\left(\begin{array}{ll}
1 & \gamma \\
0 & 1
\end{array}\right), \quad \gamma \equiv 2 \frac{\beta}{\alpha} \neq 0
$$

In order to guarantee that the fixed point of $f^{2}$ near $\eta$ is parabolic - and not more degenerate - we need only check that the map in local coordinates,

$$
f^{2}(x, y)=(x+\gamma y+p(x, y), y+q(x, y)),
$$


satisfies the inequality $\partial^{2} q / \partial x^{2} \neq 0$ at the fixed point. For our case this quantity is the following

$$
\frac{\partial^{2} q}{\partial x^{2}}(0,0)=\alpha \frac{\partial^{2} G_{1}}{\partial x^{2}}(0,0)-\alpha^{2} \frac{\partial^{2} V_{1}}{\partial u^{2}}(0,0) .
$$

By inversion of $f_{1}$ we obtain

$$
\frac{\partial^{2} V_{1}}{\partial u^{2}}(0,0)=-\frac{1}{\alpha} \frac{\partial^{2} G_{1}}{\partial x^{2}}(0,0)
$$

therefore we arrive at

$$
\frac{\partial^{2} q}{\partial x^{2}}(0,0)=2 \alpha \frac{\partial^{2} G_{1}}{\partial x^{2}}(0,0)
$$

which is nonzero as required, due to the quadratic tangency of $\operatorname{Fix}(S)$ and $f(\operatorname{Fix}(S))$ at $\xi$.

\section{Orbit Bounds}

In this appendix, we obtain a sufficient condition for the forward orbit of a point under the lift $F$ of the map (1) to be unbounded. This condition is used in the proof of Th. 2.

Write the lift as

$$
\left(\begin{array}{l}
x_{t+1} \\
y_{t+1}
\end{array}\right)=A\left(\begin{array}{l}
x_{t} \\
y_{t}
\end{array}\right)+\hat{g}\left(x_{t}\right)\left(\begin{array}{l}
1 \\
1
\end{array}\right)
$$

where $A$ is the matrix in (3), and $\hat{g}(x+1)=\hat{g}(x)$. The formal solution to this iteration is

$$
\left(\begin{array}{l}
x_{t} \\
y_{t}
\end{array}\right)=A^{t}\left(\begin{array}{l}
x_{0} \\
y_{0}
\end{array}\right)+\sum_{j=0}^{t-1} \hat{g}\left(x_{t-1-k}\right) A^{j}\left(\begin{array}{l}
1 \\
1
\end{array}\right) .
$$

The $t^{t h}$ power of the Anosov matrix (3) is easily computed in terms of the Fibonacci sequence,

$$
F_{t+1}=F_{t}+F_{t-1}, \quad F_{-2}=1, \quad F_{-1}=0,
$$

to obtain

$$
A^{t}=\left(\begin{array}{cc}
F_{2 t} & F_{2 t-1} \\
F_{2 t-1} & F_{2 t-2}
\end{array}\right)
$$

Thus (25) becomes

$$
\left(\begin{array}{l}
x_{t} \\
y_{t}
\end{array}\right)=A^{t}\left(\begin{array}{l}
x_{0} \\
y_{0}
\end{array}\right)+\sum_{j=0}^{t-1} \hat{g}\left(x_{t-1-j}\right)\left(\begin{array}{c}
F_{2 j+1} \\
F_{2 j}
\end{array}\right) .
$$

Supposing that $\|\hat{g}(x)\|_{\infty}=G$, we can find a lower bound on the orbit as

$$
\begin{aligned}
& x_{t} \geq F_{2 t} x_{0}+F_{2 t-1} y_{0}-G \sum_{j=0}^{t-1} F_{2 j+1}, \\
& y_{t} \geq F_{2 t-1} x_{0}+F_{2 t-2} y_{0}-G \sum_{j=0}^{t-1} F_{2 j} .
\end{aligned}
$$


The solution to the Fibonacci difference equation (26) is

$$
F_{t}=\frac{\phi+2}{5}\left[\phi^{t}+(-\phi)^{-t-2}\right] \geq \frac{\phi+2}{5}\left(\phi^{t}-1\right),
$$

where $\phi$ is the golden mean. Thus

$$
\begin{aligned}
\sum_{j=0}^{t-1} F_{2 j+1} & =\frac{\phi+2}{5}\left[\phi^{2 t}-1+\phi^{-2}\left(\phi^{-2 t}-1\right)\right] \leq \frac{\phi+2}{5} \phi^{2 t} \\
\sum_{j=0}^{t-1} F_{2 j} & =\frac{\phi+2}{5}\left[\phi^{2 t-1}-\phi^{-2 t-1}\right] \leq \frac{\phi+2}{5} \phi^{2 t-1}
\end{aligned}
$$

Consequently if $x_{0}, y_{0} \geq 0$, then

$$
\begin{aligned}
& x_{t} \geq \frac{\phi+2}{5}\left[\left(\phi^{2 t}\left(x_{0}+\phi^{-1} y_{0}-G\right)-x_{0}-y_{0}\right],\right. \\
& y_{t} \geq \frac{\phi+2}{5}\left[\left(\phi^{2 t-1}\left(x_{0}+\phi^{-1} y_{0}-G\right)-x_{0}-y_{0}\right] .\right.
\end{aligned}
$$

Therefore, whenever

$$
x_{0}+\phi^{-1} y_{0}>G, \quad x_{0}, y_{0}>0,
$$

then we have $x_{t}, y_{t} \rightarrow \infty$ as $t \rightarrow \infty$.

For the form (6) with $\mu=\mu_{p}(\varepsilon)$ from (9), the sup-norm of $\hat{g}$ is

$$
G=\frac{1}{2 \pi}\left[\sqrt{\varepsilon(1+\varepsilon)}+1+\varepsilon-\sec ^{-1}(1+\varepsilon)\right] \leq \frac{1}{2 \pi}(2 \varepsilon+1) .
$$

Thus the forward orbit of a point $\left(x_{0}, y_{0}\right)$ in the positive quadrant that satisfies

$$
x_{0}+\phi^{-1} y_{0}>\frac{1}{2 \pi}(2 \varepsilon+1)
$$

is unbounded.

\section{Actions and Areas}

Areas bounded by segments of invariant manifolds of an exact, area-preserving map $F: \mathbb{R}^{2} \rightarrow \mathbb{R}^{2}$ can be computed using the action-flux formulas of MacKay, Meiss, and Percival [MMP84, MMP87, Mei92]. In particular, suppose that $F$ preserves the area form $\omega$, i.e., $F^{*} \omega=\omega$, and $\omega=-d \nu$ is an exact form. We say that $F$ is exact, area-preserving when there exists a zero-form $L: M \rightarrow \mathbb{R}$ such that

$$
F^{*} \nu-\nu=d L
$$

In particular, the lift of (1) is exact symplectic with form $\omega=d x \wedge d y$ with the Lagrangian

$$
L(x, y)=\frac{1}{2}(y+g(x))^{2}+G(x)
$$

where $G$ is any anti-derivative of $g$. 
Suppose that $z^{*}=\left(x^{*}, y^{*}\right)$ is a hyperbolic or parabolic fixed point of $F$ and $\mathcal{U}=W^{u}\left(z^{*}, \zeta\right)$ is the segment of the right-going unstable manifold between $z^{*}$ and the point $\zeta=(\xi, \eta) \in W^{u}\left(z^{*}\right)$. Let $\zeta_{t}=F^{t}(\zeta)$ denote points on the orbit of $\zeta=\zeta_{0}$, so that $\zeta_{t} \rightarrow z^{*}$ as $t \rightarrow-\infty$.

Consider the region $\mathcal{R}$ "below" the segment $\mathcal{U}$ and above $x$-axis, as sketched in Fig. 16 . This region is bounded by the loop

$$
\partial \mathcal{R}=\left\{(x, 0): x^{*} \leq x \leq \xi\right\}+\{(\xi, y): 0 \leq y \leq \eta\}-\mathcal{U}-\left\{\left(x^{*}, y\right): 0<y<y^{*}\right\} .
$$

The area of $\mathcal{R}$ is

$$
A^{u}=\int_{\mathcal{R}} \omega=-\oint_{\partial \mathcal{R}} \nu=\int_{\mathcal{U}} \nu
$$

upon doing the trivial integrals along the straight segments of $\partial \mathcal{R}$. The remaining integral of the one-form $\nu$ along the segment $\mathcal{U}$ can be done using (29), recursion, and the fact that $F^{-t}(\mathcal{U}) \rightarrow z^{*}$ :

$$
\begin{aligned}
\int_{\mathcal{U}} \nu & =\int_{F^{-1}(\mathcal{U})} d L+\int_{F^{-1}(\mathcal{U})} \nu=L\left(\zeta_{-1}\right)-L\left(z^{*}\right)+\int_{F^{-1}(\mathcal{U})} \nu \\
& =\sum_{t=-\infty}^{-1}\left(L\left(\zeta_{t}\right)-L\left(z^{*}\right)\right) \equiv \Delta \mathcal{A}^{-}\left(\zeta, z^{*}\right),
\end{aligned}
$$

the difference between the past actions of the two orbits.

For the map (1), the upper half of the channel $\tilde{E}^{+}(\xi)$ has boundary (17). Since the fixed points have $y^{*}=0$, the channel area is the difference between the areas below the hyperbolic manifold and that below the parabolic manifold, as given by (31):

$$
A^{+}(\xi)=\operatorname{Area}\left(E^{+}(\xi)\right)=\Delta \mathcal{A}^{-}\left(\zeta_{h}, h\right)-\Delta \mathcal{A}^{-}\left(\zeta_{p}, p\right)
$$

Areas computed using this formula for the map (1) are shown in Fig. 10.

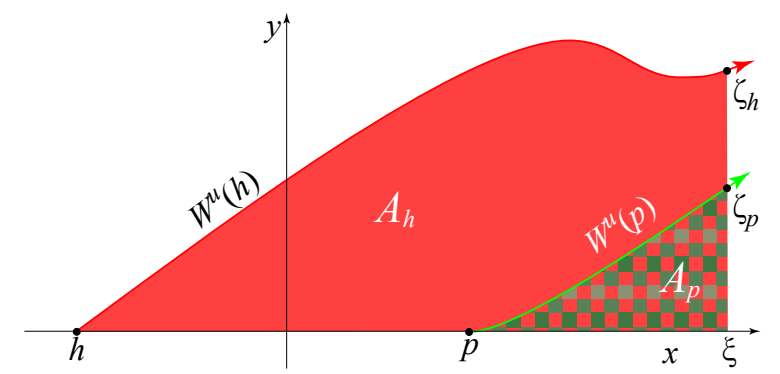

Figure 16: Areas below the initial segments of the right-going unstable manifolds of $\left(x_{h}, 0\right)$ (red), and $\left(x_{p}, 0\right)$ (green checkerboard) for $\varepsilon=1.0$.

For a symmetric fixed point $z^{*}$, the reversor, (7), maps $S\left(W^{u}\left(z^{*}\right)\right)=W^{s}\left(z^{*}\right)$. The image of the channel $\tilde{E}^{+}$is bounded by the curve

$$
S\left(\partial \tilde{E}^{+}\right)=\left\{(x, 0): x_{h} \leq x \leq \xi\right\}+W^{s}\left(p, S\left(\zeta_{p}\right)\right)+\left\{(\xi-y, y):-\eta_{p} \geq y \geq-\eta_{h}\right\}-W^{s}\left(h, S\left(\zeta_{h}\right)\right)
$$


Note that the reflected channel has a cut-off that is a line segment with slope minus one. Now since

$S$ is area-preserving, but orientation reversing, the area of the stable channel $\tilde{E}^{-}=S\left(\tilde{E}^{+}\right)$is

$$
\int_{\tilde{E}^{-}} \omega=\oint_{\partial \tilde{E}^{-}} \nu=-\oint_{S\left(\partial \tilde{E}^{+}\right)} \nu=-\int_{\tilde{E}^{+}} \omega .
$$

Thus, up to the sign, the areas are the same.

\section{References}

[AA90] S. Aubry and G. Abramovici. Chaotic trajectories in the standard map, the concept of anti-integrability. Physica D, 43:199-219, 1990. http://dx.doi.org/10.1016/ 0167-2789 (90) 90133-A.

[ALD83] S. Aubry and P.Y. Le Daeron. The discrete Frenkel-Kontorova model and its extensions. Physica D, 8:381-422, 1983. http://dx.doi.org/10.1016/0167-2789(83)90233-6.

[Arn63] V.I. Arnold. Small denominators and problems of stability of motion in classical and celestial mechanics. Russ. Math. Surveys, 18:6:85-191, 1963. http://dx.doi.org/10. 1070/RM1963v018n06ABEH001143.

[Ber78] M.V. Berry. Regular and irregular motion. In S. Jorna, editor, Topics in Nonlinear Dynamics : A Tribute to Sir Edward Bullard, volume 46 of AIP Conf. Proc., pages 16-120. AIP, New York, 1978. http://dx.doi.org/10.1063/1.31417.

[BM46] S. Bochner and D. Montgomery. Locally compact groups of differentiable transformations. Ann. of Math. (2), 47(4):639-653, 1946. http://www.jstor.org/stable/ 1969226.

[Boc02] J. Bochi. Genericity of zero Lyapunov exponents. Ergod. Theory Dynam. Syst., 22:16671696, 2002. http://dx.doi.org/10.1017/S0143385702001165.

[CE01] E. Catsigeras and H. Enrich. SRB measures of certain almost hyperbolic diffeomorphisms with tangency. Discr. Cont. Dynam. Systet., ser. A, 7(1):177-202, 2001. http://dx. doi.org/10.3934/dcds.2001.7.177.

[Dua08] P. Duarte. Elliptic isles in families of area-preserving maps. Ergodic Theory and Dynamical Systems, 28(06):1781-1813, 2008. http://dx.doi.org/10.1017/ S0143385707000983.

[Fon99] E. Fontich. Stable curves asymptotic to a degenerate fixed point. Nonlinear Anal: Theory, Methods, Appl., 35:711-733, 1999. http://dx.doi.org/10.1016/S0362-546X(98) 00004-2.

[Fra70] J. Franks. Anosov diffeomorphisms. In S.-S. Chern and S. Smale, editors, 1970 Global Analysis, volume XIV of Proc Symp. on Pure Math, pages 61-93. Am. Math. Soc, 1970. 
[GK82] M. Gerber and A. Katok. Smooth models of Thurston's pseudo-Anosov maps. Ann. Scient. Éc. Norm. Super., ser.4, 15:173-204, 1982. http://www.numdam.org/item?id= ASENS_1982_4_15_1_173_0.

[Gor12] A. Gorodetski. On stochastic sea of the standard map. Comm. Math. Phys., 309(1):155192, 2012. http://dx.doi.org/10.1007/s00220-011-1365-z.

[Gri77] V.Z. Grines. The topological conjugacy of diffeomorphisms of a two-dimensional manifold on one-dimensional orientable basic sets. II. Trans. Am. Math. Soc., 1978(2):243-252, 1977. (translation of Trudy Moskov. Mat. Obšč. 34:243-252, 1977.).

[GTS07] S. Gonchenko, D. Turaev, and L. Shilnikov. Homoclinic tangencies of arbitrarily high orders in conservative and dissipative two-dimensional maps. Nonlinearity, 20:241-275, 2007. http://dx.doi.org/10.1088/0951-7715/20/2/002.

[HPS77] M.W. Hirsch, C. Pugh, and M. Shub. Invariant Manifolds, volume 583 of Lecture Notes in Mathematics. Springer-Verlag, New York, 1977.

[Kat79] A.K. Katok. Bernoulli diffeomorphisms on surfaces. Ann. Math., 110:529-547, 1979. http://dx.doi.org/10.2307/1971237.

[Ler10] L. Lerman. Breaking hyperbolicity for smooth symplectic toral diffeomorphisms. Regul. Chaotic Dyn., 15(2-3):194-209, 2010. http://dx.doi.org/10.1134/ S1560354710020085.

[Lew80] J. Lewowicz. Lyapunov functions and topological stability. J. Differential Equations, 38:192-209, 1980. http://dx.doi.org/10.1016/0022-0396(80)90004-2.

[Liv04] C. Liverani. Birth of an elliptic island in a chaotic sea. Math. Phys. Electron. J., 10:1-13, 2004. http://www.maia.ub.es/mpej/Vol/10/1.pdf.

[LR98] J.W.S. Lamb and J.A.G. Roberts. Time-reversal symmetry in dynamical systems: A survey. Physica D, 112:1-39, 1998. http://dx.doi.org/10.1016/S0167-2789(97) 00199-1.

[Mac93] R.S. MacKay. Renormalisation in Area-Preserving Maps, volume 6 of Adv. Series in Nonlinear Dynamics. World Scientific, Singapore, 1993.

[Mar69] N.G. Markley. The Poincaré-Bendixon theorem for the Klein bottle. Trans. A.M.S., 135:159-165, 1969. http://dx.doi.org/10.2307/1995009.

[Mat82] J.N. Mather. Existence of quasi-periodic orbits for twist homeomorphisms of the annulus. Topology, 21:457-467, 1982. http://dx.doi.org/10.1016/0040-9383(82)90023-4.

[Mei92] J.D. Meiss. Symplectic maps, variational principles, and transport. Reviews of Modern Physics, 64(3):795-848, 1992. http://dx.doi.org/10.1103/RevModPhys.64.795.

[MMP84] R.S. MacKay, J.D. Meiss, and I.C. Percival. Transport in Hamiltonian systems. Physica D, 13:55-81, 1984. http://dx.doi.org/10.1016/0167-2789(84)90270-7. 
[MMP87] R.S. MacKay, J.D. Meiss, and I. C. Percival. Resonances in area-preserving maps. Physica D, 27(1-2):1-20, 1987. http://dx.doi.org/10.1016/0167-2789(87)90002-9.

[New77] S.E. Newhouse. Quasi-elliptic periodic points in conservative dynamical systems. Am. J. Math., 99:1061-1087, 1977. http://dx.doi.org/10.2307/2374000.

[Prz82] F. Przytycki. Examples of conservative diffeomorphisms of the two-dimensional torus with coexistence of elliptic and stochastic behaviour. Ergod. Th. Dyn. Sys., 2:439-463, 1982. http://dx.doi.org/10.1017/S0143385700001711.

[Sin95] Ya. Sinai. A mechanism of ergodicity in the standard map. In C. Simo, editor, Hamiltonian Systems with Three or More Degrees of Freedom, volume 533 of Series C: Mathematical and Physical Sciences, pages 242-243. NATO ASI, 1995. http: //dx.doi.org/10.1007/978-94-011-4673-9.

[Sma67] S. Smale. Differentiable dynamical systems. Bull. Am. Math. Soc., 73:747-817, 1967. http://www. ams.org/journals/bull/1967-73-06/S0002-9904-1967-11798-1/.

[Wei36] A. Weil. Les familles de courbes sur le tore. Mat. Sborn., Nov. Ser., 1(5):779-781, 1936. 